\title{
A particle finite element based model for droplet spreading analysis
}

Elaf Mahrous, ${ }^{1, \text { a) }}$ Alex Jarauta, 2, b) Thomas Chan, ${ }^{3}$ Pavel Ryzhakov, ${ }^{4,5, \text { c) }}$ Adam Z. Weber, ${ }^{3}$ R. Valéry Roy, ${ }^{1, d)}$ and Marc Secanell 6, e)

${ }^{1)}$ Department of Mechanical Engineering, University of Delaware, Newark, DE 19716,

USA

${ }^{2)}$ Energy Systems Design Lab (ESDLab), University of Alberta, Edmonton, AB T6G 2G8,

Canada

${ }^{3)}$ Energy Conversion Group, Lawrence Berkeley National Laboratory, Berkeley, CA 94720,

USA

4) Universitat Politècnica de Catalunya, BarcelonaTech, 08034 Barcelona, Spain

${ }^{5)}$ Centre Internacional de Mètodes Numèrics a I'Enginyeria (CIMNE), 08034 Barcelona,

Spain

${ }^{6}$ Corresponding author; Energy Systems Design Lab (ESDLab), University of Alberta, Edmonton, AB T6G 2G8, Canada

(Dated: 28 April 2020)

A particle finite element method (PFEM) based model is proposed to analyze droplet dynamics problems, particularly droplet spreading on solid substrates (wetting). The model uses an updated Lagrangian framework to formulate the governing equations of the liquid. Curvature of the liquid surface is tracked accurately using a deforming boundary mesh. In order to predict the spreading rate of the droplet on the solid substrate and track the corresponding contact angle evolution, dissipative forces at the contact line are included in the formulation in addition to the Navier-slip boundary conditions at the solid-liquid interface. The inclusion of these boundary conditions makes it possible to account for the induced Young's stress at the contact line and for the viscous dissipation along the solid-liquid interfacial region. These are found to be essential to obtain a mesh-independent physical solution. The temporal evolution of the contact angle and contact line velocity of the proposed model are compared with spreading droplets and micro sessiledroplet injection experiments, and are shown to be in good agreement.

Keywords: Droplet spreading; Surface tension; Dissipative forces; Young's stress; Wetting; Capillary wavelength; PFEM

\section{INTRODUCTION}

Droplet dynamics modeling is an active area of research in the computational fluid dynamics (CFD) community due to the vast number of associated practical applications, such as inkjet printing ${ }^{112}$, cooling towers ${ }^{3}$, and water transport in fuel cell porous media 4 and gas channels ${ }^{910}$. In these applications, characterized by dominant capillary forces, the liquid phase is found in contact with solid substrates that can be hydrophobic, hydrophilic, or chemically heterogeneous ${ }^{11}$. Droplet dynamics models typically encounter several challenges when studying these phenomena: a) tracking of the free liquid surface ${ }^{12-14}$, b) identifying the interaction forces between liquids and substrates ${ }^{15}-18$, and hence tracking the transition between inertial and viscoelastic regimes 1920 , and c) obtaining mesh-dependent solutions $51-24$.

For sessile droplet problems involving contact between a liquid and a solid substrate, Young's stress is induced at the contact line as the droplet spreads 22 . This leads to a special slip regime where the contact line velocity evolves proportionally to the dynamic contact angle 15 . The classical Navier-Stokes equation alone does not account for the contact

\footnotetext{
a)Electronic mail: mahrouse@udel.edu

b)Electronic mail: jarautaa@ualberta.ca

c)Electronic mail: pryzhakov@cimne.upc.edu

${ }^{\mathrm{d})}$ Electronic mail: vroy@udel.edu

e)Electronic mail: secanell@ualberta.ca
}

line motion of the liquid on a no-slip substrate and therefore cannot relieve the moving contact line singularity, which leads to nonphysical velocity evolution and energy dissipation known as "Huh and Scriven's paradox, 2226-292. For droplets and thin films on hydrophilic surfaces, lubrication approximation theory can be used as a valid simplified and cost-efficient numerical model to regularize the contact line singularity 25 . However, this theory is not valid for hydrophobic surfaces and pinned contact line analysis. Thus, more sophisticated models are needed for analyzing droplet dynamics (droplet deformation, pinning, and spreading) in the case of hydrophobic and chemically heterogeneous substrates. For such cases, the contact line singularity can be relieved by introducing i) a slip zone at the contact line, that accounts for the normal and tangential stresses, and the capillary effect due to the contact line motion, and ii) a slip zone away from the contact line, that accounts for shear and viscous stresses acting inside the solidliquid interfacial region $14 \mid 15 / 2230$.

The simplest attempt to resolve the singularity at the contact line consists in applying a static contact angle condition where the direction of the vector normal to the liquid-air surface at the contact line is constant and equal to the value of the equilibrium contact angle for the considered substrate $31+33$. However, this approach cannot account for droplet pinning, and therefore, cannot predict the dynamics of spreading droplets. A dynamic contact angle condition is needed to account for this phenomenor 934 . The most basic dynamic contact line condition imposes a slip boundary condition, i.e., contact line velocity normal to wall surface is zero, when a critical con- 
tact angle is reached 16 . This condition implies that no energy is dissipated as the contact line moves on the solid substrate, and that the velocity of the contact line is not restricted, which contradicts experimental observations 35 .

In order to achieve a physically relevant contact line velocity, several authors proposed a contact line boundary condition that depends on a dissipative force term. Spel ${ }^{36}$ proposed a linearly dependent contact angle condition that is a function of the sign of the contact line velocity. Manservisi and Scardovelli 30 added a dissipative, resistive force applied to the contact line as a function of the capillary number. Buscaglia and Ausas ${ }^{22}$ presented a variational formulation of a surface tension model that included a dissipative force acting on the contact line that was proportional to the velocity of the contact line; however, their model did not account for contact line pinning, which often takes place on rough substrates. In addition to the dissipative force condition, Ren and $\mathrm{E}^{[15}$ observed that the normal stress inside the solid-liquid interface exhibits a large jump across the contact line, which varies linearly with its velocity, and hence should be balanced and considered as an additional boundary condition. Moreover, Venkatesan et $a l{ }^{16}$ observed that the tangential stress at the contact line is proportional to its tangential velocity, which was included as an additional term in their numerical formulation.

Obtaining a mesh-independent solution is an additional modeling challenge. Several authors have studied the dependence of the numerical solution of wetting phenomena on the mesh size. In reference ${ }^{30}$, Manservisi and Scardovelli studied the spreading behavior of droplets by utilizing the Lagrangian front tracking approach, and concluded that adding the dissipative energy term reduces the spreading rate until a nearly no-slip condition is achieved. Afkhami et al. ${ }^{21}$ conducted a numerical study using the Volume-of-fluid (VOF) method to analyze the dependence of the dynamic contact angle evolution on the mesh size. They observed that this dependence could be reduced by relating the dynamic contact angle to the capillary number and to the mesh size. More recently, Buscaglia and Ausas2 2 introduced a variational formulation and analyzed the effect of adding dissipative forces as constant values on the mesh dependency of the solution. They concluded that increasing the dissipative force term leads to a less mesh-dependent solution. In addition, Venkatesan et al. ${ }^{16}$ used an Arbitrary Lagrangian-Eulerian (ALE) finite element formulation and introduced a slip coefficient in the Navierslip term that is a function of the mesh size, Weber number, and Reynolds number. They managed to alleviate the spurious mesh dependency. However, they observed that the proposed relation did not work properly for hydrophobic surfaces.

Even though a substantial effort has been invested in developing robust and efficient numerical models for contact line dynamics of sessile droplets, a physically accurate and mesh-independent model does not currently exist to the best of our knowledge. In addition, most of the aforementioned models were applied to fixed-mesh methods. Our previous work has shown that a moving-grid particle finite element method (PFEM)-based model is well suited to droplet modeling 910/34/37]. Application of a PFEM model has proven to be particularly advantageous when dealing with surface tension dominated problems, as it allows to accurately track the evolution of the liquid domain boundary and to account for surface tension, without introducing enrichment shape functions or smearing the surface tension via continuous force approach 3839. However, neither a dissipative force term on the contact line nor a viscous dissipation term on the solid-liquid interface has been implemented and tested in PFEM-based droplet models. The objective of this article is to identify an appropriate dynamic contact line condition for the PFEM-based fluid flow solver proposed in ${ }^{34 / 37}$ and to verify that it provides a mesh-independent solution. In the present work, our proposed droplet dynamic model is equipped with i) an effective slip contact angle boundary condition that balances the induced Young's stress, and ii) a solid-liquid slip boundary condition, away from the contact line, that accounts for the viscous dissipation along the solid-liquid interface ${ }^{15 / 22}$.

The article is organized as follows. Section $\mathrm{II}$ includes a description of the moving grid model for droplet dynamics. Special emphasis is given to the boundary conditions applied at the solid-liquid interface including the contact line. Numerical results are shown in Section III] including a detailed analysis of the manner in which dissipative forces can produce a less mesh-dependent solution. The model is validated by comparing numerical results for droplet spreading on hydrophilic and hydrophobic surfaces with experimental data. We performed further experimental validation with a droplet injection setup, which includes two different smooth substrates.

\section{PHYSICAL MODEL}

\section{A. Governing equations}

Let us consider a domain $\Omega$ which represents a liquid droplet in contact with a solid substrate, as shown in Fig. 1. The boundary of the domain is split into three regions $\partial \Omega=$ $\Gamma_{I} \cup \Gamma_{S} \cup \partial \Gamma$. The part of the domain boundary corresponding to the liquid in contact with a substrate, excluding the contact line, is designated as $\Gamma_{S}$. The contact line is denoted by $\partial \Gamma$. The rest of the boundary of the liquid phase is denoted by $\Gamma_{I}$, which corresponds to the droplet free surface. For twodimensional problems, the contact line reduces to two triple points. The droplet is assumed to be surrounded by its saturated liquid-vapor phase 40 . It is assumed that the velocity of the vapor is negligible, i.e., it exerts no mechanical action upon the droplet and thus the droplet can be analyzed as an isolated entity.

The governing equations for the liquid phase are the momentum and mass conservation equations s1142 $^{\text {? The fluid is }}$ considered incompressible, viscous, and Newtonian. Thus, the governing equations read $12 \mid 34$.

$$
\begin{array}{ll}
\rho\left(\frac{\partial \boldsymbol{v}}{\partial t}+(\boldsymbol{c} \cdot \nabla) \boldsymbol{v}\right)-\nabla \cdot(2 \mu D \boldsymbol{v}-p \mathbb{I})=\rho \boldsymbol{g} & \text { on } \Omega \\
\nabla \cdot \boldsymbol{v}=0 & \text { on } \Omega
\end{array}
$$

where $\rho$ is the fluid density, $v$ is velocity, $t$ is time, $\mu$ is the fluid dynamic viscosity, $D \boldsymbol{v}=\left(\nabla \boldsymbol{v}+\nabla^{T} \boldsymbol{v}\right) / 2$ is the strain rate 


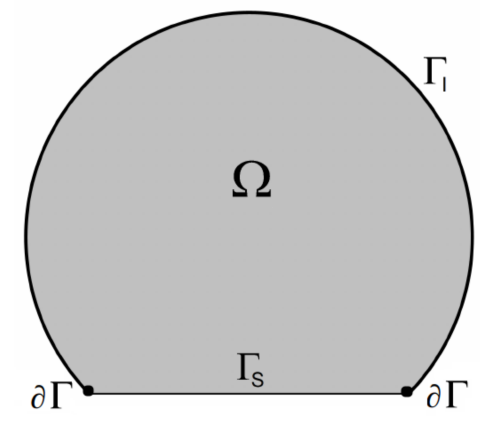

FIG. 1. Schematic representation of the considered Lagrangian domain at the continuum level.

tensor, $p$ is pressure, $\mathbb{I}$ is the identity tensor, and $\boldsymbol{g}$ is the gravitational acceleration. The convective velocity is the relative velocity between the material and the mesh, $\boldsymbol{c}=\boldsymbol{v}-\boldsymbol{v}_{m}$, where $\boldsymbol{v}_{m}$ is the mesh velocity. In the present model the convective velocity is zero because the Lagrangian reference frame is adopted ${ }^{43}$, and therefore the fluid and mesh velocities are the same.

At the interface $\Gamma_{I}$, a Cauchy stress boundary condition in the normal direction of the stress is applied corresponding to the surface tension force:

$$
f_{\Gamma_{I}}=\boldsymbol{\sigma} \cdot \boldsymbol{n}=\gamma \kappa \boldsymbol{n} \quad \text { at } \Gamma_{I}
$$

where $f_{\Gamma_{I}}$ is the surface tension force, $\sigma$ is the Cauchy stress tensor, $\boldsymbol{n}$ is the outer unit normal to $\Gamma_{I}, \gamma$ is the surface tension coefficient, and $\kappa$ is the curvature of the interface. According to Eq. (3), the normal stress is balanced by the surface tension force $10[2]$. 37 .

At the solid-liquid interface excluding the contact line, $\Gamma_{S}$, the applied boundary condition corresponds to the shear stresses in order to account for viscous dissipation. It is obtained by projecting the Cauchy stress tensor on the normal direction of $\Gamma_{S} 15 \mid 22$ :

$$
f_{\Gamma_{S}}=\boldsymbol{\sigma} \cdot \boldsymbol{n}=-\beta_{\Gamma_{s}} \boldsymbol{v} \quad \text { at } \Gamma_{S}
$$

where $f_{\Gamma_{S}}$ and $\beta_{\Gamma_{s}}$ are the dissipative force and the slip coefficient applied at the solid-liquid interface, respectively, and $v$ is the slip velocity of the fluid on the solid-liquid interface. A variety of models have been proposed in the literature for the slip coefficient, $\beta_{\Gamma_{s}}$, at the solid-liquid interface such as, Navier-slip condition $\left(\beta_{s}\right)^{14]}$, prescribed slip profile condition 15 , and a constant slip coefficient that depends on the grid size 22 . The Navier-slip model is considered in this work, as it accounts for the shear rates and viscous dissipation along the solid-liquid interface during droplets deformation 14 1621.

At the contact line, an effective slip boundary condition is applied corresponding to the total dissipative force, including the contribution of i) the capillary effect $(\zeta)$, ii) normal stress coefficient $\left(\beta_{n}\right)$, and iii) Navier-slip coefficient $\left(\beta_{s}\right)$, and is proportional to the velocity of the contact line ${ }^{14} 1622$ :

$$
\boldsymbol{f}_{\partial \Gamma}=-\beta_{\partial \Gamma} \boldsymbol{v} \quad \text { at } \partial \Gamma
$$

where $f_{\partial \Gamma}$ is the dissipative force applied at the contact line, $\beta_{\partial \Gamma}$ is the effective slip coefficient at the contact line, and $v$ is the slip velocity of the fluid at the contact line.

The details on the dissipative forces applied at the contact line and the solid-liquid interface are presented next.

\section{B. Forces acting at the contact line, $\partial \Gamma$}

Let us consider a steady droplet in contact with a flat surface forming a static contact angle $\theta_{s}$ (Fig. 2). Under this condition, the equilibrium is expressed as the balance of the liquid surface tension $\left(\gamma_{l v}\right.$, or simply denoted by $\left.\gamma\right)$, solid surface energy $\left(\gamma_{s v}\right)$, and interfacial tension $\left(\gamma_{s l}\right)$. When normalized to a unit length, these forces are the interfacial tensions between the three phases (solid/liquid/vapor). By projecting the equilibrium forces on the solid plane, one obtains the well known Young's equation $45 \mid 46$.

$$
\gamma_{l v} \cos \theta_{s}=\gamma_{s v}-\gamma_{s l}
$$

The contact line velocity that corresponds to this equilibrium state is $v=0$. In this case, the boundary condition applied to the contact line is the following: if the contact angle is within a given range of values, i.e., $\theta \in\left[\theta_{\min }, \theta_{\max }\right]$, the contact line is fixed. A fixed contact line is usually referred to as a pinned contact line. The values of minimum and maximum contact angle to achieve contact line pinning, which depend on both the liquid and the substrate, are determined experimentally 911 .

As the droplet starts to spread, Eq. (6) does not hold any longer and therefore a slip boundary condition is applied for the velocity at the contact line $\partial[9,47$.

$$
\boldsymbol{v} \cdot \boldsymbol{n}=0
$$

This condition, however, results in an unrealistic contact angle evolution because the velocity of the contact line is not restricted by the physical viscous dissipation at the solidliquid interface, leading to a non-physical spreading of the moving contact line. Instead, according to 1522 , the velocity of the contact line can be related to the Young's stress acting on it:

$$
u \propto \gamma\left(\cos \theta_{s}-\cos \theta\right)
$$

where $u$ is the tangential component of the velocity vector at the contact line, i.e., $u=v \cdot t$, and $\theta$ is the dynamic contact angle. The proportionality coefficient between the contact line velocity and Young's stress is the effective slip boundary condition defined as follows 15 .

$$
\beta_{\partial г} u=\gamma\left(\cos \theta_{s}-\cos \theta\right)
$$

where $\beta_{\partial \Gamma}$ is the effective slip coefficient, and is defined

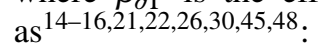

$$
\beta_{\partial \Gamma}=\zeta+\beta_{s}+\beta_{n}
$$




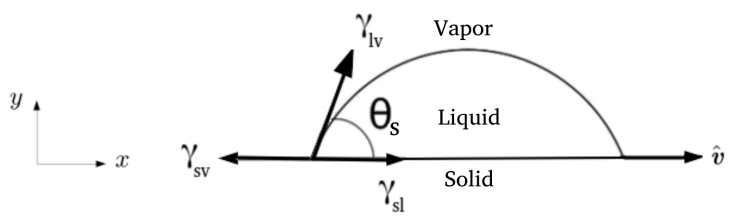

FIG. 2. Schematic representation of the forces acting on a contact line of a sessile droplet.

where $\zeta$ is the dynamic capillary effect coefficient, $\beta_{s}$ is the Navier-slip coefficient, and $\beta_{n}$ is the normal stress coefficient.

The capillary effect coefficient is expressed as follows $22[30$ :

$$
\zeta=\frac{\gamma}{u} R(C a)
$$

where $R(\mathrm{Ca})$ is a function of the capillary number calculated from the contact line velocity 30 . In several experimental studies, expressions for $R(\mathrm{Ca})$ were obtained by fitting empirical data. Among these studies, the models proposed by Jiang 49 , Bracke 50 , and Seeberg are commonly used 51 . The numerical and dynamic contact angle simulations in reference ${ }^{52}$ concluded that Jiang's model was able to predict higher capillary flow velocities among these three empirical models. For capillary-driven spreading droplet, the contact line velocity is of the order of an impact velocity of a droplet ${ }^{35 \mid 53}$; therefore, Jiang's expression is used in this work, i.e., 49 .

$$
R(C a)=\left(\cos \theta_{s}+1\right) \tanh \left(4.96 C a^{0.702}\right)
$$

where $\theta_{s}$ is the static contact angle, and $C a=u \mu / \gamma$ is the capillary number calculated using the contact line velocity. Thus, the coefficient $\zeta$ in Eq. (11) is expressed as follows:

$$
\zeta=\frac{\gamma}{u}\left(\cos \theta_{s}+1\right) \tanh \left(4.96 C a^{0.702}\right)
$$

The Navier-slip coefficient, $\beta_{s}$ in Eq. (10), is a function of the shear stress, and it is calculated using 14 161 .

$$
\beta_{s}=\frac{1}{u} \mu \nabla(\boldsymbol{v} \cdot \boldsymbol{t}) \cdot \boldsymbol{n}
$$

In addition, larger jump in normal stresses is introduced across the contact line as its velocity increases 15 . The normal stress jump across the contact line is balanced by including the coefficient $\beta_{n}$, which is expressed as follows 15 .

$$
\beta_{n}=\frac{1}{u} \mu \nabla(\boldsymbol{v} \cdot \boldsymbol{t}) \cdot \boldsymbol{t}
$$

where $u$ is the tangential component of the velocity vector at the contact line, i.e., $u=v \cdot t$.

After considering the contributions of the capillary effects, the Navier-slip as well as the normal stresses in Eq. (10), the coefficient $\beta_{\partial \Gamma}$ is used to obtain the total dissipative force and to apply the boundary condition defined by Eq. (5).

\section{Forces acting at the solid-liquid interface away from the contact line, $\Gamma_{S}$}

When a viscous fluid is in contact with a solid substrate, the velocity of the fluid at the fluid-solid interface is equal to the velocity of the solid. If the solid is at rest, a no-slip boundary condition is applied in this region. In wetting problems, however, the no-slip condition contradicts the physical dissipation phenomena on the solid-liquid interfacial zone. It has been observed that introducing a Navier-slip boundary condition on droplet spreading problem partially resolves this contradiction, and also reduces the mesh-dependency of the numerical solution 14 16/21/22. Therefore, at the solid-liquid interface, $\Gamma_{S}$, the Navier-slip boundary condition is applied in the present work, according to Eq. (4). Accordingly, the slip coefficient $\beta_{\Gamma_{s}}$ at the solid-liquid interface $\Gamma_{S}$ is expressed as follows:

$$
\beta_{\Gamma_{s}}=\beta_{s}=\frac{1}{u} \mu \nabla(\boldsymbol{v} \cdot \boldsymbol{t}) \cdot \boldsymbol{n}
$$

where $u$ is the tangential component of the velocity vector at the solid-liquid interface. Eq. (16) is used in combination with Eq. (4) to apply the boundary condition at the solid-liquid interface.

\section{Mesh size and time step criteria}

As the droplet spreads, capillary waves are generated at the contact line and move toward the free surface, i.e., from $\partial \Gamma$ towards $\Gamma_{1} 54 \mid 55$. For a spreading droplet, the maximum spreading velocity is of the order of the droplet impact velocity $35 \mid 56$. At this velocity, Yuriko et al ${ }^{57}$ concluded that the capillary wave is observed when $W e>1$, where the Weber number defined as:

$$
W e=\frac{\rho R u_{\max }^{2}}{\gamma}
$$

where $\rho$ is the liquid density, $R$ is the droplet radius, and $u_{\text {max }}$ is the maximum value of the tangential component of the velocity vector at the contact line. The latter value, $u_{\max }$, can be estimated experimentally, and it is known to depend on both the fluid and the substrate 35 56. In this article, different values of $u_{\max }$ are used based on the experimental scenarios illustrated in Section

Yuriko et al $\frac{57}{5}$ also observed that the capillary wavelength, $\lambda$, corresponding to the contact line capillary propagation is estimated by:

$$
\lambda=\frac{\gamma}{\rho u_{\text {max }}^{2}}
$$

Numerically, the mesh size at the contact line is restricted by the wavelength of the capillary wave propagation, and its maximum value $h$ can be estimated to be 59 :

$$
h=\frac{\lambda}{2}
$$


Substituting Eq. 18) into Eq. (19) yields:

$$
h=\frac{\gamma}{2 \rho u_{\max }^{2}}
$$

Moreover, the wave velocity corresponding to the dominant capillary wavelength is calculated as 58160 .

$$
c=\sqrt{\frac{2 \pi \gamma}{\rho \lambda}}
$$

where $c$ is the wave velocity. The critical time step corresponding to this capillary wave, $\Delta t_{\text {cap }}$, can also be estimated as 59 :

$$
\Delta t_{c a p}=\frac{1}{2 f} \leq \frac{h}{c}
$$

where $f$ is the maximum frequency in $\mathrm{s}^{-1}$ corresponding to the capillary wave propagation at the contact line. Substituting Eq. 21] and Eq. 201 into Eq. 22, yields:

$$
\Delta t_{\text {cap }} \leq \sqrt{\frac{2}{\pi}} \frac{\gamma}{4 \rho\left|u_{\max }^{3}\right|}
$$

Ryzhakov et al. ${ }^{6162}$ suggested the time step to be used for the PFEM approach should scale to half of the critical time step in order to avoid element degradation or inversion, which yields:

$$
\Delta t \leq \sqrt{\frac{2}{\pi}} \frac{\gamma}{8 \rho\left|u_{\max }^{3}\right|}
$$

\section{E. Discretized governing equations}

The particle finite element method (PFEM) $\sqrt{38 \mid 4363+67}$ is used in the present work to discretize Eqs. (1) and (2) in space. The PFEM adopts the updated Lagrangian description of the governing equations. The fluid domain is discretized by a standard finite element mesh. The nodes can be interpreted as immaterial particles that move according to their convective velocity. At each solution step the domain deforms. Thus, in order to avoid excessive mesh degradation re-meshing is performed at each time step using Delaunay triangulation. For droplet dynamics problems re-meshing procedure ensures that the domain boundaries are preserved. Linear interpolation functions for both velocity and pressure are used over 3 -noded triangles in $2 \mathrm{D}$. In the present implementation the discretization in time is performed using the Newark-Bossak scheme. However, in the present section, it is illustrated using the Backward-Euler scheme for sake of simplicity ${ }^{43}$. A more complete algorithm for a two-phase flow model can be found in 3437 . The problem statement is the following: given a known velocity and pressure $\overline{\boldsymbol{v}}_{n}$ and $\bar{p}_{n}$ at time $t_{n}$, find the values of these variables at the next time step $t_{n+1}\left(\overline{\boldsymbol{v}}_{n+1}\right.$ and $\bar{p}_{n+1}$ ) by solving:

$$
\begin{aligned}
& \boldsymbol{M} \frac{\overline{\boldsymbol{v}}_{n+1}-\overline{\boldsymbol{v}}_{n}}{\Delta t}+\mu \boldsymbol{L} \overline{\boldsymbol{v}}_{n+1}+\boldsymbol{G} \bar{p}_{n+1}=\overline{\boldsymbol{F}}+\overline{\boldsymbol{F}}_{s t}+\overline{\boldsymbol{F}}_{\partial \Gamma}+\overline{\boldsymbol{F}}_{\Gamma_{S}} \\
& \boldsymbol{D} \overline{\boldsymbol{v}}_{n+1}=0
\end{aligned}
$$

where $\boldsymbol{M}$ is the mass matrix, $\Delta t$ is the time step, $\boldsymbol{L}$ is the Laplacian matrix, $\boldsymbol{G}$ is the gradient matrix, $\overline{\boldsymbol{F}}$ is the vector of external forces, $\overline{\boldsymbol{F}}_{s t}$ is the surface tension force vector, $\overline{\boldsymbol{F}}_{\partial \Gamma}$ is the dissipative force vector acting at the contact line, $\overline{\boldsymbol{F}}_{\Gamma_{S}}$ is the dissipative force vector acting at the solid-liquid interface away from the contact line, and $\boldsymbol{D}$ is the divergence matrix. Local cell matrices and vectors are defined in Appendix A.

The dependence on the unknown configuration, $\boldsymbol{X}_{n+1}$, introduces a geometrical nonlinearity that is solved using a Newton method. The governing equations are first written in their residual form:

$\overline{\mathbf{r}}_{m}=\overline{\boldsymbol{F}}+\overline{\boldsymbol{F}}_{s t}+\overline{\boldsymbol{F}}_{\partial \Gamma}+\overline{\boldsymbol{F}}_{\Gamma_{S}}-\left(\boldsymbol{M} \frac{\overline{\boldsymbol{v}}_{n+1}-\overline{\boldsymbol{v}}_{n}}{\Delta t}+\mu \boldsymbol{L} \overline{\boldsymbol{v}}_{n+1}+\boldsymbol{G} \bar{p}_{n+1}\right)$

$\overline{\mathbf{r}}_{c}=\boldsymbol{D} \overline{\boldsymbol{v}}_{n+1}$

Eqs. (27) and (28) are then linearized, and the system is solved iteratively. The resulting linearized system of governing equations reads:

$$
\left(\begin{array}{cc}
\boldsymbol{M} \frac{1}{\Delta t}+\mu \boldsymbol{L}+\boldsymbol{H}_{s t} & \boldsymbol{G} \\
\boldsymbol{D} & 0
\end{array}\right)\left(\begin{array}{c}
\delta \overline{\boldsymbol{v}} \\
\delta \bar{p}
\end{array}\right)=\left(\begin{array}{c}
\overline{\mathbf{r}}_{m} \\
\overline{\mathbf{r}}_{c}
\end{array}\right)
$$

where $\boldsymbol{H}_{s t}$ originates from the linearization of the surface tension force term, $\overline{\boldsymbol{F}}_{s t}$. This term is responsible for the implicit treatment of the surface tension, and it allows for using large time steps. The derivation of this term, as well as the stabilization terms which have been omitted for sake of simplicity, can be found in reference ${ }^{34}$. The linear velocity-pressure elements used in our model do not fulfill the compatibility conditior ${ }^{68}$ and therefore pressure must be stabilized. In this work, the algebraic sub-grid scales (ASGS) $\sqrt{69-71}$ stabilization technique is implemented (see reference ${ }^{34}$ for details). Once the system in Eq. (29) has been solved for $\delta \bar{v}$ and $\delta \bar{p}$, the primary variables are updated according to $\overline{\boldsymbol{v}}_{n+1}^{k+1}=\overline{\boldsymbol{v}}_{n+1}^{k}+\delta \overline{\boldsymbol{v}}$ and $\bar{p}_{n+1}^{k+1}=\bar{p}_{n+1}^{k}+\delta \bar{p}$, where $k$ is the nonlinear iteration index, until convergence is achieved. As a final step, the nodal position in the moving mesh is updated according to the employed time integration scheme. In case of Backward Euler integration this yields $\boldsymbol{X}_{n+1}^{k+1}=\boldsymbol{X}_{n}+\Delta t \overline{\boldsymbol{v}}_{n+1}^{k+1}$. Solution algorithm and implementation methodology are further illustrated in Appendices B and C, respectively.

\section{RESULTS AND DISCUSSION}

\section{A. Mesh dependence study}

This example illustrates the effects of enriching the droplet model by including the dissipative forces at both the solidliquid interface and at the contact line. The impact of this 

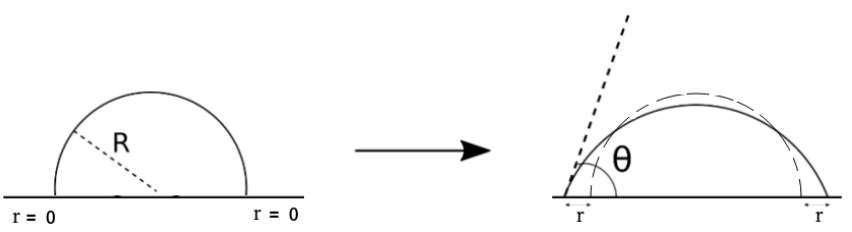

FIG. 3. Initial and steady-state configuration for a sessile droplet starting with an initial contact angle angle of $90^{\circ}$, and evolving with a spreading displacement of $r$.

enrichment upon droplet spreading, contact angle evolution, and of the mesh dependency of the solution is analyzed.

Following the experimental settings of Buscaglia and Ausas ${ }^{22}$, the geometry considered here is a sessile droplet with an initial radius of $0.125 \mathrm{~mm}$, an initial contact angle of $90^{\circ}$, and a static contact angle of $\theta_{s}=45^{\circ}$ (see Fig. 3). The driving forces acting on the droplet are due to surface tension and gravity. Fluid density, viscosity and surface tension coefficient are set to $\rho=1000 \mathrm{~kg} \mathrm{~m}^{-3}, \mu=8.90 \times 10^{-4} \mathrm{~kg} \mathrm{~m}^{-1}$ $\mathrm{s}^{-1}, \gamma=0.072 \mathrm{~N} \mathrm{~m}^{-1}$, respectively, in order to represent a water droplet. Initial pressure in the liquid is set to $p_{0}=0 \mathrm{~Pa}$.

For this study, the maximum spreading velocity at the initial stage, i.e., $t<0.25 \mathrm{~ms}$, is assumed, based on experimental analysis, to be $u_{\max } \sim 2.5 \mathrm{~m} \cdot \mathrm{s}^{-1}$. This data corresponds to a water droplet of $\sim 2 \times 10^{-4}$ m radius spreading on Triethoxysilybutraldehyde with a static concoct angle of $43^{\sqrt{53}}$. Therefore, this gives the estimations of $\lambda \sim 1.165 \times 10^{-5} \mathrm{~m}$, $c \sim 6.27 \mathrm{~m} \cdot \mathrm{s}^{-1}, h \sim 5.8 \times 10^{-6} \mathrm{~m}$, and $\Delta t=4.6 \times 10^{-7} \mathrm{~s}$ from Eq. (18), Eq. (21), Eq. (20), and Eq. (24).

Six cases are analyzed to illustrate the effect of the slip coefficient parameters at the contact line and at the solid-liquid interface excluding the contact line, $\beta_{\partial \Gamma}$ and $\beta_{\Gamma_{S}}$, as shown in table【

TABLE I. Analyzing different slip coefficient parameters at the contact line and at the solid-liquid interface excluding the contact line, $\beta_{\partial \Gamma}$ and $\beta_{\Gamma_{S}}$.

\begin{tabular}{|c|c|c|}
\hline Case number & $\beta_{\partial \Gamma}$ & $\beta_{\Gamma_{S}}$ \\
\hline Case 0 & 0 & 0 \\
\hline Case 1 & 0 & $1 \times 10^{-5} \mathrm{~Pa} \cdot \mathrm{s}$ \\
\hline Case 2 & 0 & $1 \times 10^{-3} \mathrm{~Pa} \cdot \mathrm{s}$ \\
\hline Case 3 & $1 \times 10^{-3} \mathrm{~Pa} \cdot \mathrm{s}$ & $1 \times 10^{-3} \mathrm{~Pa} \cdot \mathrm{s}$ \\
\hline Case 4 & 0 & $\beta_{s}$, using Eq. 16 \\
\hline Case 5 & $\zeta$, using Eq. 13 & 0 \\
\hline Case 6 & $\zeta+\beta_{s}+\beta_{n}$, using Eqs. 13 15 & $\beta_{s}$ using Eq. 16, \\
\hline
\end{tabular}

Figure 4 shows the predicted spreading displacement defined as $r$ in Fig. 3, and contact angle evolution when no dissipative forces are applied. To compare the general spreading behavior with Buscaglia and Ausas 22 , a time step of $\Delta t=$ $2 \times 10^{-7} \mathrm{~s}$ is used. Four different mesh sizes were adopted in order to study the solution sensitivity to mesh size, prior to adding the proposed boundary conditions (i.e., Eq. (4) and Eq. (5)), as follows: i) $h=1.25 \times 10^{-5} \mathrm{~m}$ with 340 elements, ii) $h=9.6 \times 10^{-6} \mathrm{~m}$ with 598 elements, iii) $h=6.8 \times 10^{-6}$ m with 1205 elements, and iv) $h=5 \times 10^{-6} \mathrm{~m}$ with 2206 elements. Parameter $h$ represents the minimum distance between

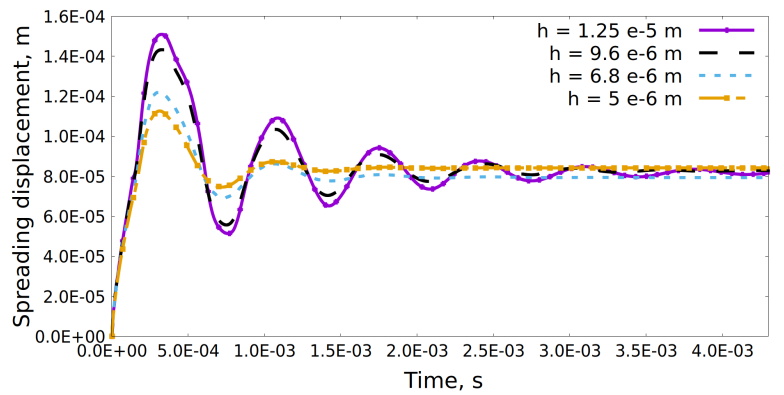

(a)

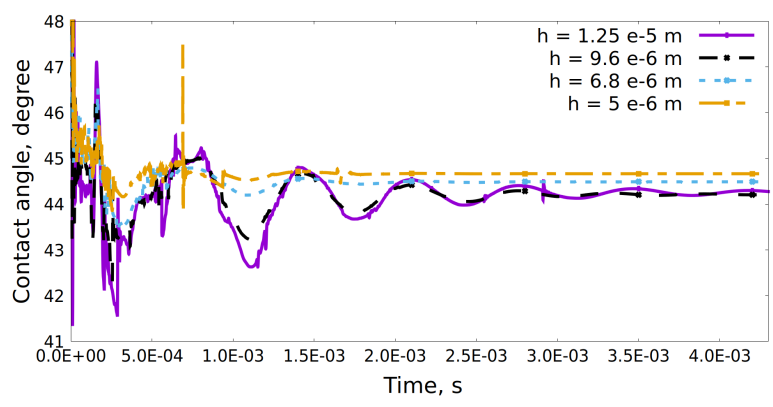

(b)

FIG. 4. Evolution of (a) spreading displacement and (b) contact angle, for $\beta_{\partial \Gamma}=0$ and $\beta_{\Gamma_{S}}=0$; case 0 in table I using different mesh sizes.

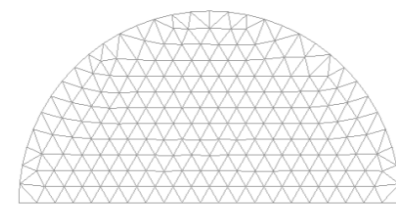

(a)

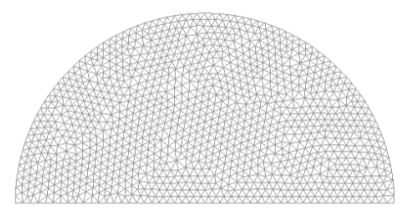

(b)
FIG. 5. Initial configuration of a sessile droplet with a starting contact angle of $90^{\circ}$ : a) coarse mesh of $1.25 \times 10^{-5} \mathrm{~m}$ and b) fine mesh of $5 \times 10^{-6} \mathrm{~m}$.

the nodes of a triangular mesh. As observed by Buscaglia and Ausas ${ }^{22}$, when no dissipative force boundary conditions are applied, the solution is mesh-dependent, and the contact angle evolution is highly oscillatory.

The coarsest and the finest meshes, i.e., $h=1.25 \times 10^{-5}$ $\mathrm{m}$ and $h=5 \times 10^{-6} \mathrm{~m}$, are considered for further study. These are displayed in Fig 5. For each mesh size, the six above-mentioned sets of dissipative forces and slip conditions were included.

Fig. 6) shows spreading displacement and contact angle evolution for coarse mesh simulations. The absence of the dissipative term $(\zeta)$, normal stress coefficient $\left(\beta_{n}\right)$, or the Navierslip coefficient $\left(\beta_{s}\right)$ causes an oscillatory spreading behavior and contact angle evolution (Fig. 6(a), Fig. 6(c)) . Experimental results 355356 do not show this oscillatory behavior for a fluid on hydrophilic substrates. Therefore, these spu- 


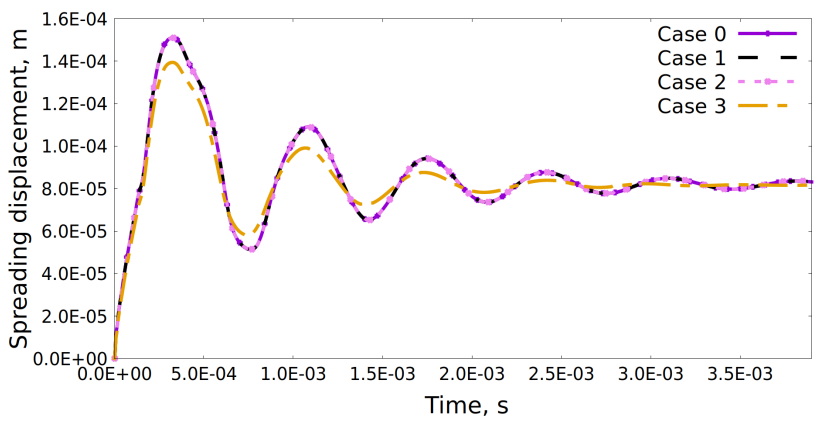

(a)

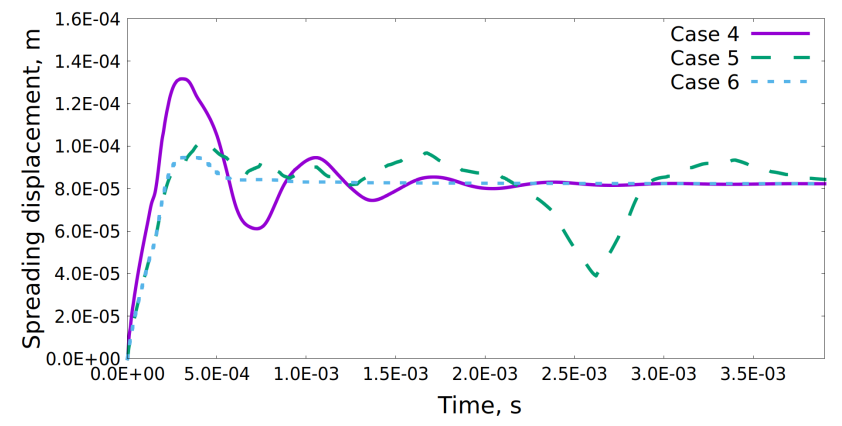

(b)

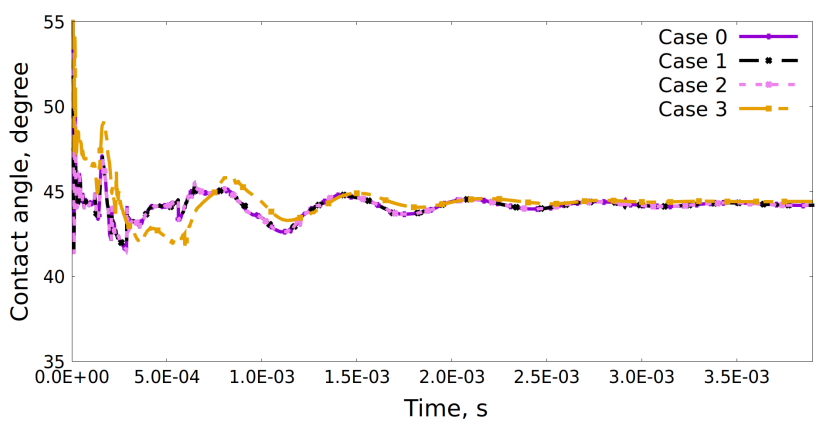

(c)

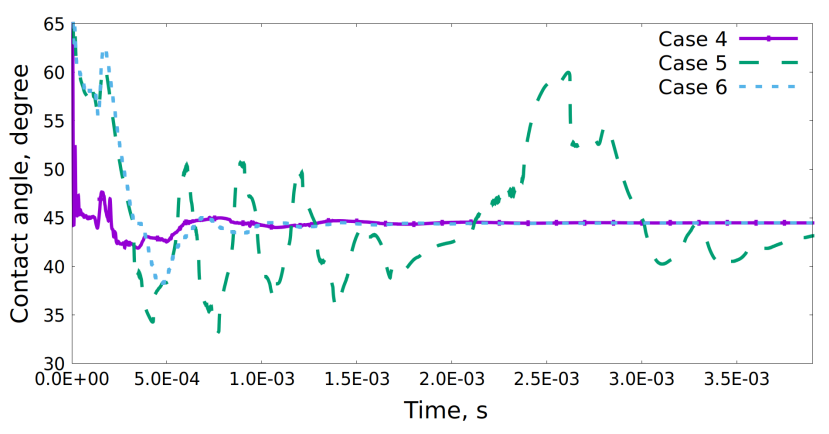

(d)

FIG. 6. Evolution of spreading displacement for constant $\beta_{\partial \Gamma}$ and $\beta_{\Gamma_{S}}$ (a) and using Eq. (10) and (16) (b). Contact angle evolution for constant $\beta_{\partial \Gamma}$ and $\beta_{\Gamma_{S}}(\mathrm{c})$, and using Eq. 10) and 16 (d), see table I Mesh size of $h=1.25 \times 10^{-5} \mathrm{~m}$ (coarse mesh). rious oscillations are hypothesized to be due to the numerical scheme ${ }^{2230}$. The initial non-physical spreading evolution causes the contact line to exceed its equilibrium contact angle, i.e., $\theta_{d}<45^{\circ}$. Hence, the contact line retracts toward its equilibrium state again. The contact line spreads back and forward in a manner proportional to the induced Young's stress (Eq. (8) until its effect vanishes and the droplet reaches its equilibrium condition.

The first two cases show that increasing the constant value of the Navier-slip coefficient at the solid-liquid interface, $\beta_{\Gamma_{S}}$, does not guarantee a physical spreading evolution. Adding a constant slip coefficient at the contact line, i.e., case 3, reduces the contact angle and spreading rate oscillatory behavior, and increases the convergence rate. Yet, these constant values do not produce physical contact angle and spreading evolution rates.

Adding the Navier-slip condition on $\Gamma_{S}$ (Eq. [16), case 4) reduces the contact line singularity and enhances the convergence rate towards the equilibrium condition (Fig. 6(b) and Fig. 6(d)). However, the contact angle continues to behave in a non-physical manner due to the absence of the dynamic capillary term $(\zeta)$.

Adding the dynamic capillary term alone, case 5, at the contact line (Eq. (13) ) results in unstable contact angle evolution and spreading rate (Fig. 6(b) and Fig. 6(d)). This is interpreted by the absence of the physical viscous dissipation and stress balance terms acting on the solid-liquid interface, including the contact line.

Adding the dissipative force terms at the contact line and at the solid-liquid interface ( $\beta_{\partial \Gamma}$ and $\beta_{\Gamma_{S}}$, respectively) using Eq. (10), and (16) enhances the overall behavior of both contact angle and contact line evolution (Fig. 6(b) and Fig. 6(d)). The contact line reaches its equilibrium state after $0.0006 \mathrm{~s}$, i.e $\approx 85 \%$ faster than without resorting to the proposed boundary conditions; the non-physical oscillatory spreading behavior vanishes. Furthermore, case 6 in Fig. 6(b) shows two physical zones. The first zone is the inertial zone, $0<t<4 \times 10^{-4} \mathrm{~s}$, where the droplet spreads at a rate proportional to the induced Young's stress. The second zone is the viscous zone, $t>4 \times 10^{-4} \mathrm{~s}$, where the viscous dissipation is dominating as the droplet reaches its equilibrium contact angle $19|35| 53 \mid 56$.

The proposed boundary conditions at the solid-liquid interface are critical to achieving physically relevant droplet spreading solutions with the use of coarse meshes. Results for the refined mesh, i.e., $h=5 \times 10^{-6} \mathrm{~m}$, are displayed in Fig. 7 In the absence of the dissipative term, normal stress coefficient, and Navier-slip coefficient, a smoother spreading displacement evolution is achieved, compared to the coarser mesh (Fig. 7(a) and Fig. 6(a). Furthermore, the equilibrium state is reached after $0.0012 \mathrm{~s}$. However, a non-physical oscillatory contact angle temporal evolution with low amplitudes is observed in Fig. 7(c) A more refined mesh leads to a lower nodal velocity and slip length coefficient, $l_{\beta}$, at the vicinity of the contact line compared to a coarse mesh 1672 . Accordingly, a higher slip coefficient, $\beta_{s}$, is imposed in the Navier-slip for- 


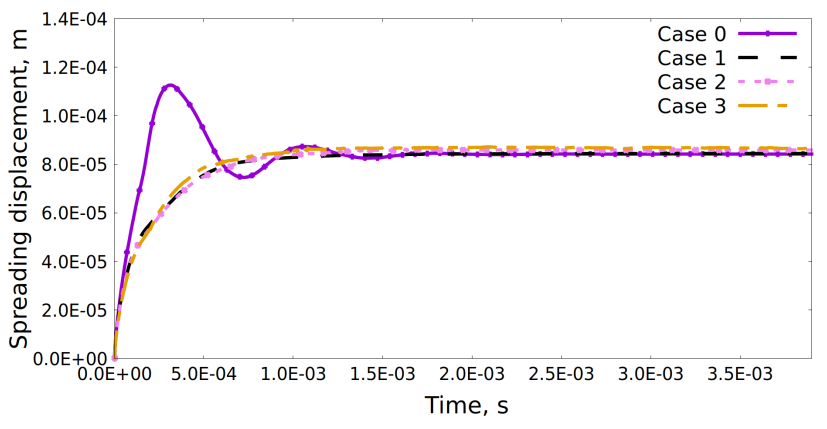

(a)

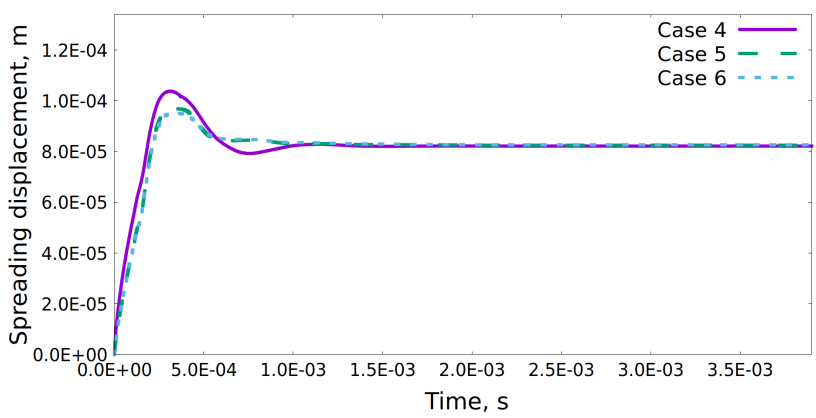

(b)

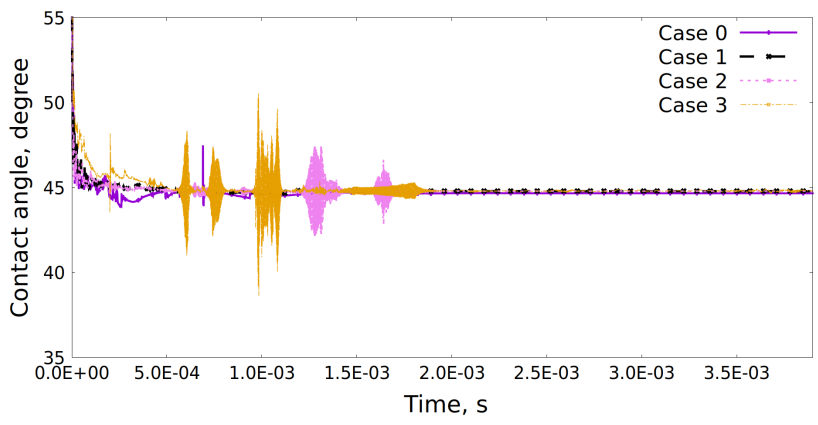

(c)

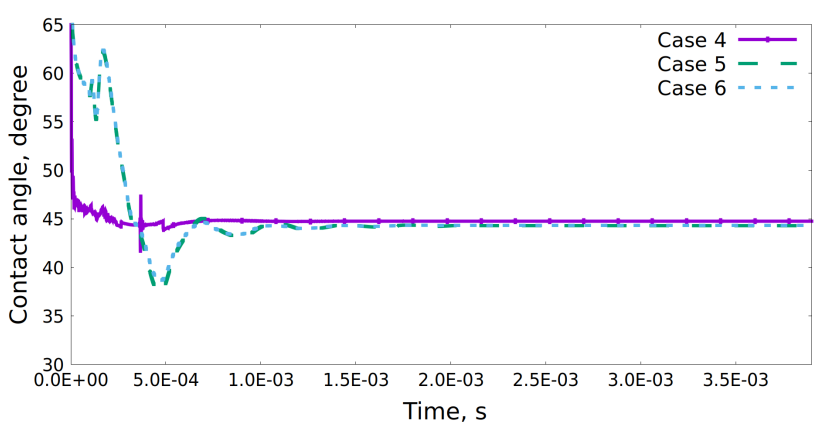

(d)

FIG. 7. Evolution of spreading displacement for constant $\beta_{\partial \Gamma}$ and $\beta_{\Gamma_{S}}$ (a) and using Eq. (10) and (16) (b). Contact angle evolution for constant $\beta_{\partial \Gamma}$ and $\beta_{\Gamma_{S}}(\mathrm{c})$, and using Eq. 10) and 16) (d), see table I Mesh size of $h=5 \times 10^{-6} \mathrm{~m}$ (fine mesh). mulation, such that ${ }^{2122}$ :

$$
\beta_{s}=\frac{\mu}{l_{\beta}}
$$

where $\mu$ is the fluid dynamic viscosity. On the one hand, the use of more refined mesh results in a smoother contact angle evolution and spreading behavior. On the other hand, the oscillatory behavior of the contact angle evolution is due to the missing dissipative force term that controls the contact angle evolution as a function of the dynamic capillary effect. Adding the capillary effect, $\zeta$, at contact line, case 5 and case 6 , results in smooth physical transition between the inertial and viscous regions for both spreading displacement and contact angle evolution.

The contact angle evolution and spreading rate for both mesh sizes are compared before and after applying the complete set of proposed boundary conditions at the solid-liquid interface and contact line are shown in Fig. 8. Results obtained without imposing the dissipative force boundary conditions are grid dependent and disply large, non-physical oscillations. In contrast, the results obtained with the numerical solver with the proposed boundary conditions are grid independent and nearly free of oscillations with a smooth physical transition between both the inertial regime, $0<t<2 \times 10^{-4} \mathrm{~s}$, and the viscous regime, $t>5 \times 10^{-4} \mathrm{~s}$. These results demonstrate that the proposed boundary conditions enhance the stability of the contact line motion and achieve a mesh independent solution with larger element sizes.

\section{B. Comparison with experimental data}

In order to assess the validity of the proposed numerical model, we compare in this section experimental and numerical results of several studies involving spreading of fluids on flat smooth surfaces with varying contact angles. Specifically, the following cases are studied:

1. Spreading of a water droplet on a hydrophobic substrate, i.e., Triethoxysilane, (with equilibrium contact angle of $117.0^{\circ} \sqrt{53}$.

2. Spreading of a squalane $\left(\mathrm{C}_{30} \mathrm{H}_{62}\right)$ droplet on a silica substrate (with equilibrium contact angle of $38.8^{\circ}$ ) 35 .

3. Spreading of water on Teflon (hydrophobic) and kapton (hydrophillic) substrates (with equilibrium contact angles of $108^{\mathrm{d73}}$ and $75^{\sqrt{74}}$, respectively).

The first two studies are based on published experimental studies, while the third case is based on experiments performed by the authors.

\section{Hydrophobic substrate}

The proposed model is used to simulate the sessile water droplet deposition experiments performed by Bird et al ${ }^{[53}$. In 


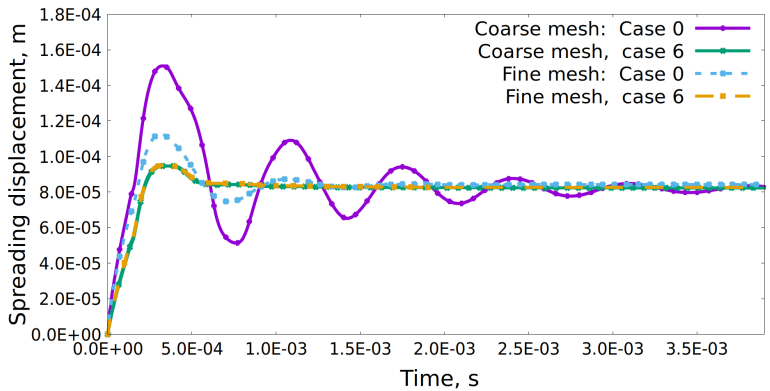

(a)

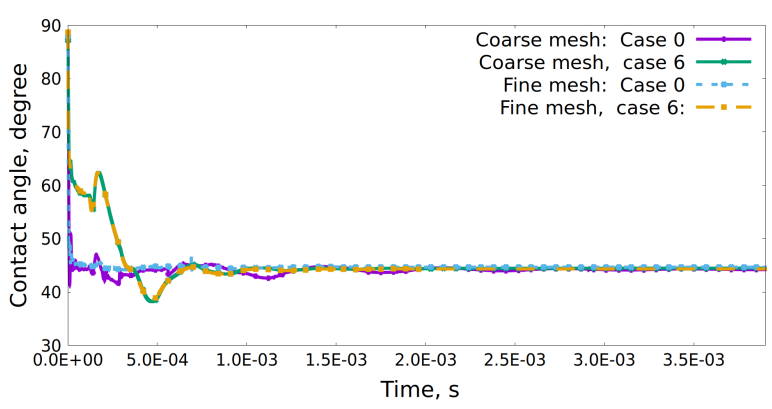

(b)

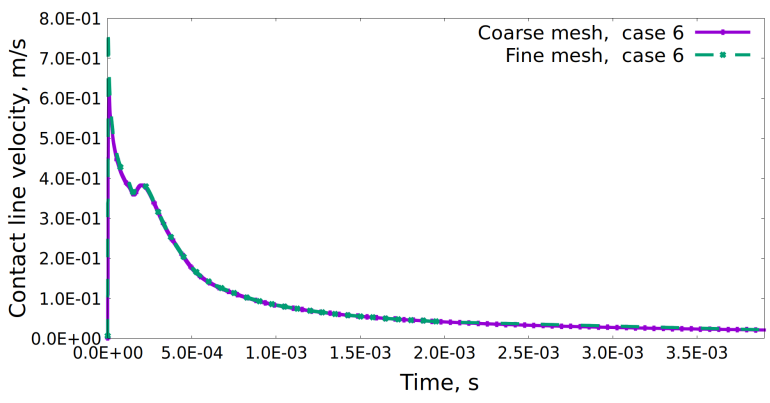

(c)

FIG. 8. Less mesh-dependent results for (a) spreading displacement evolution, (b) contact angle temporal evolution, and (c) contact line velocity evolution, using our proposed boundary conditions and for two different triangular mesh sizes of $h=1.25 \times 10^{-5} \mathrm{~m}$ (coarse mesh) and $h=5 \times 10^{-6} \mathrm{~m}$ (fine mesh).

this study, a drop was placed on the Triethoxysilane substrate through a needle. The drop contacts the substrate at a diameter of $1 \mathrm{~mm}$, and was allowed to spread spontaneously over the substrate until equilibrium was reached, i.e., until the value $\theta=\theta_{s}=117^{\circ}$ is reached. Spreading displacement (r) was measured over time during the initial time of wetting.

In order to reproduce this experiment, a droplet is placed on the substrate with an initial radius $R=5 \times 10^{-4} \mathrm{~m}$, contact angle of $180^{\circ}$, and liquid pressure of $p_{0}=0 \mathrm{~Pa}$ (Fig. 9). Then, the droplet is allowed to deform until equilibrium is achieved, i.e., $\theta_{s}=117^{\circ}$. Fluid density, viscosity and surface tension coefficient are set to $\rho=1000 \mathrm{~kg} \mathrm{~m}^{-3}, \mu=8.90 \times 10^{-4} \mathrm{~kg}$ $\mathrm{m}^{-1} \mathrm{~s}^{-1}, \gamma=0.072 \mathrm{~N} \mathrm{~m}^{-1}$, respectively. Assuming that the capillary waves at the contact line are dominants and $u_{\max }$ of the order $\sim 1 \mathrm{~m} \mathrm{~s}^{-153}$, two simulations were performed using

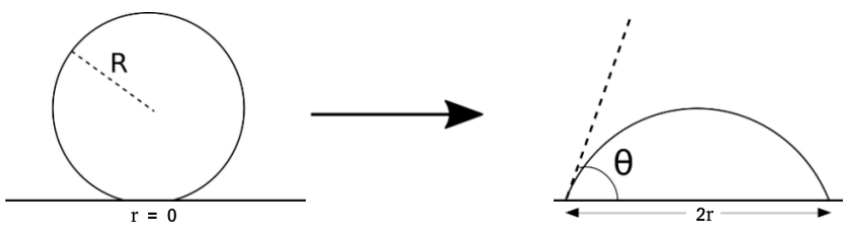

FIG. 9. Initial and steady-state configuration for the sessile droplet starting with an initial contact angle $\approx 180^{\circ}$, and evolving with a spreading displacement of $r$.
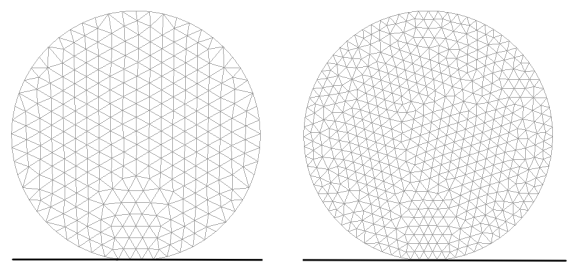

FIG. 10. Two different mesh sizes, $h=8 \times 10^{-5} \mathrm{~m}$ and $h=4 \times 10^{-5}$ $\mathrm{m}$, representing the initial-stage of $0.001 \mathrm{~m}$ diameter droplet.

the boundary conditions of Eq. (10) and $(16)$, two element sizes, i.e., $h=4 \times 10^{-5} \mathrm{~m}$ with 1085 elements, and $h=8 \times$ $10^{-5} \mathrm{~m}$ with 282 elements, as displayed in Fig. 10 , and a timestep size of $10^{-7} \mathrm{~s}$.

Fig. 11] shows the spreading profile within the first millisecond when the initial capillary wave propagation is dominating at the contact line and moving toward the free surface. The profile obtained numerically are in good agreement with the experimental profile ${ }^{53}$. Fig. 12 shows the spreading displacement, and spreading rate evolution observed experimentally and predicted numerically using the two meshes. Experimental and numerical results are in very good agreement, and the two meshes provide very similar results, i.e., the numerical results are mesh independent. The initial contact line velocity reaches $1 \mathrm{~m} \mathrm{~s}^{-1}$, which corresponds to the predicted velocity regime for an impact or spreading droplet starting from res ${ }^{35156}$. Fig. 12 and Fig. 11(a) show that the numerical model is able to capture the early spreading regime, $t<0.1 \mathrm{~ms}$ where the spreading rate is independent of the wettability of the substrate ${ }^{53 / 75 / 76}$. Furthermore, the inertial regime characterized by the capillary waves ${ }^{19 \mid 77}$ is captured numerically. This can be verified based on the transition time interval, $T_{t}$, formula between the inertial dominated regime, $T_{i}$, and the viscous dominated regime, $T_{v}$, i.e., $T_{i}<T_{t}<T_{v}$, such that 1977678 .

$$
\left(\frac{\rho R^{3}}{\gamma}\right)^{0.5}<T_{t}<\left(\frac{\rho R^{3}}{\gamma}\right)^{0.5}\left(\frac{\rho \gamma R}{\mu^{2}}\right)^{0.125}
$$

where $T_{t}$ is the transition time interval between the inertial and the viscous regimes, $\rho$ is the fluid density, $R$ is the initial droplet radius, $\gamma$ is the surface tension coefficient, and $\mu$ is the dynamic viscosity. Eq. (31) implies that the inertial regime for a water droplet of radius $0.5 \mathrm{~mm}$ is in the range of $T_{i}<1.3 \mathrm{~ms}$, after which the transition regime takes place. Finally, Fig 11 


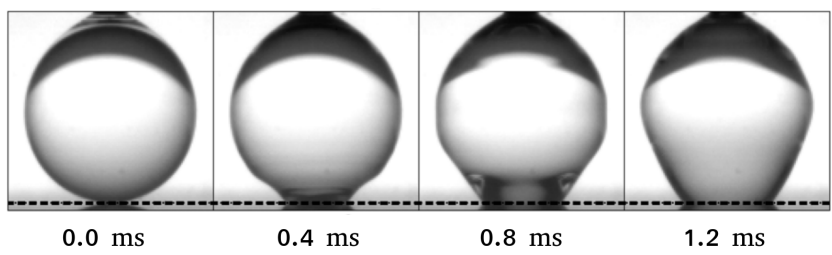

(a) Water droplet of a radius $0.82 \pm 0.01 \mathrm{~mm}$.

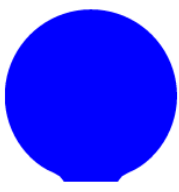

$0.1 \mathrm{~ms}$

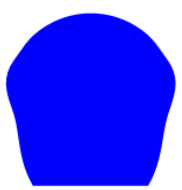

$0.6 \mathrm{~ms}$

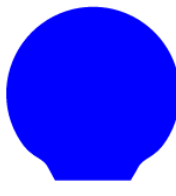

$0.2 \mathrm{~ms}$

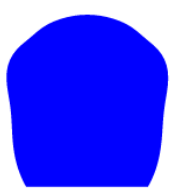

$0.7 \mathrm{~ms}$

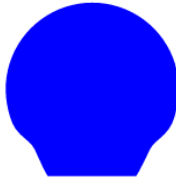

$0.3 \mathrm{~ms}$

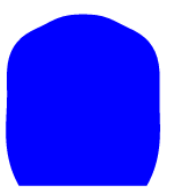

$0.8 \mathrm{~ms}$
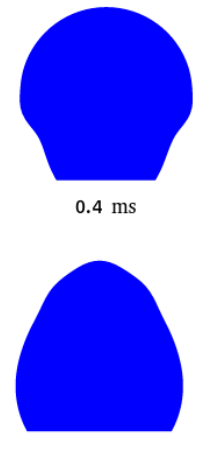

$1.2 \mathrm{~ms}$ (b) Water droplet of a radius $0.5 \mathrm{~mm}$.

FIG. 11. Water droplet spreading on Triethoxysilane, for $0<t \leq 1.2$ $\mathrm{ms}$, with a dominant capillary wave propagation at the contact line (a) experimentally, (reproduced with permission from J. Phys. Rev. Lett. 100, 234501 (2008). Copyright 2008 American Physical Society), and (b) numerically.

shows that the spreading regime depends on the initial droplet radius, i.e., the smaller the droplet initial radius the faster the transition from inertial to viscous regimes, in agreement with Eq. (31).

\section{Hydrophilic substrate}

In this section, the numerical model is used to reproduce the experimental results obtained by Seveno et al ${ }^{[35]}$ where squalane is allowed to spread over a silica substrate. The static contact angle of squalane on silica is $38.8^{\circ}$.

To reproduce this experiment the same initial and boundary conditions are used as in the previous case, as shown in Fig. 9. The initial droplet diameter is $0.0018 \mathrm{~m}$ and the physical properties of the fluid are $\rho=810 \mathrm{~kg} \mathrm{~m}^{-3}, \mu=0.0314 \mathrm{~kg}$ $\mathrm{m}^{-1} \mathrm{~s}^{-1}$, and $\gamma=0.0311 \mathrm{~N} \mathrm{~m}^{-1}$. The expected spreading rate is of the order $10^{-1} \mathrm{~m} \frac{35}{\text {. Assuming }} u_{\text {max }}=0.5 \mathrm{~m} \mathrm{~s}^{-1}$, the capillary wavelength and corresponding mesh sizes are obtained by Eq. (18) and Eq. 20) as $\lambda=1.5 \times 10^{-4} \mathrm{~m}$ and $h=7.510^{-5}$ $\mathrm{m}$ with 1014 elements, respectively. Also, since $W e>1$ as per Eq. (17), the capillary wave propagation should be expected from numerical model. The time step is chosen as $\Delta t=10^{-7}$ $\mathrm{s}$, according to Eq. 24 covering the range of contact line velocities of the order $10^{-1} \mathrm{~m} \mathrm{~s}^{-1}$.

Fig. 13 shows the contact angle evolution versus the contact line velocity, followed by the contact angle evolution ver-

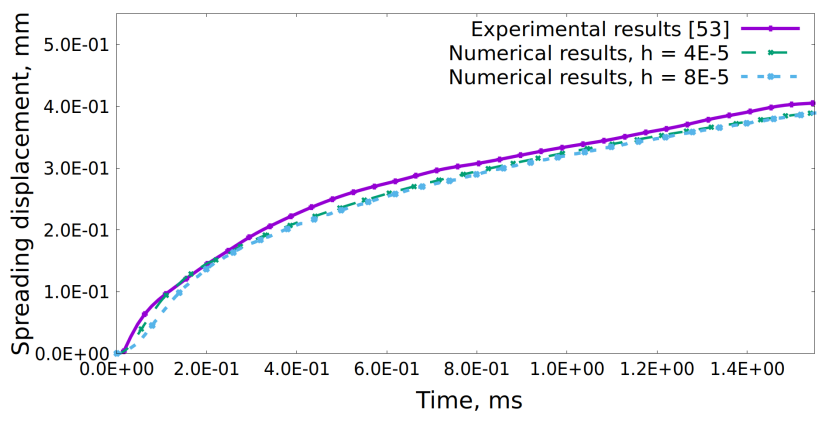

(a)

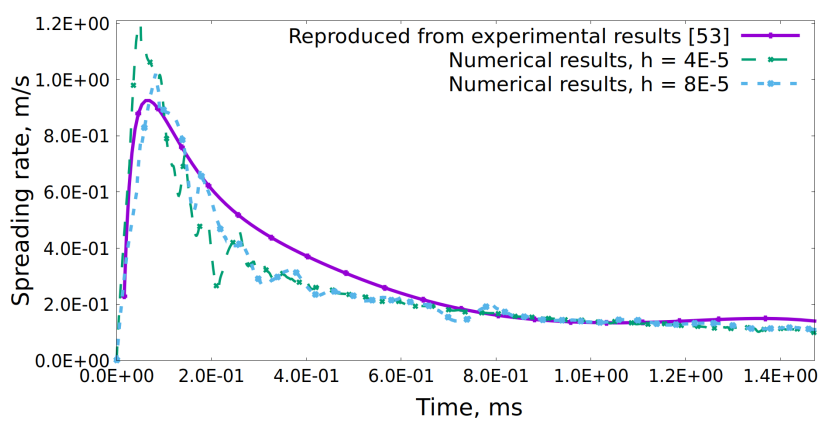

(b)

FIG. 12. Water droplet spreading rate on Triethoxysilane: (a) spreading displacement evolution (numerically vs. experimentally) and (b) contact line velocity evolution (numerically vs. experimentally).

sus time. The numerically predicted contact line velocity evolution is in agreement with measured experimental data (see Fig. 13(a) and is proportional to the temporal evolution of the contact angle (Fig. 13(b)). Furthermore, the initial squalane droplet velocity on silica is of the order of $\sim 0.1 \mathrm{~m} \mathrm{~s}^{-1}$ (Fig. 13(a) as indicated by Seveno et al. ${ }^{35}$. Both numerical and experimental results show that the contact line spreading is initially dominated by inertial effects, followed by a reduction of the contact line velocity, where viscous effects dominate. This observation is in agreement with Eq. (31), where the transition time interval between the inertial and the viscous regimes is predicted as, $4.3 \times 10^{-3} \mathrm{~s}<T_{t}<6.4 \times 10^{-3}$ s. In this time interval, the contact angle value varies between approximately $150^{\circ}$ and $125^{\circ}$ (Fig 13(b). Therefore, the numerical oscillatory behavior in this region, as shown in Fig 13(a) is hypothesized to be due to the transition between the inertial and the viscous regimes.

\section{Droplet injection experiments}

To further validate the proposed model, water injection experiments on hydrophillic (Kapton, static contact angle of $75^{\text {(74) }}$ ) and hydrophobic (PTFE, static contact angle of 108 ${ }^{\mathrm{c73}}$ ) substrates were performed by the authors. In the experiment, water was injected from a small hole of $6.0 \mathrm{~mm}$ diameter in the substrate using a peristaltic pump at two constant flow rates, i.e., $5 \mu \mathrm{L} / \mathrm{s}$ and $10 \mu \mathrm{L} / \mathrm{s}$. Three experiments were per- 


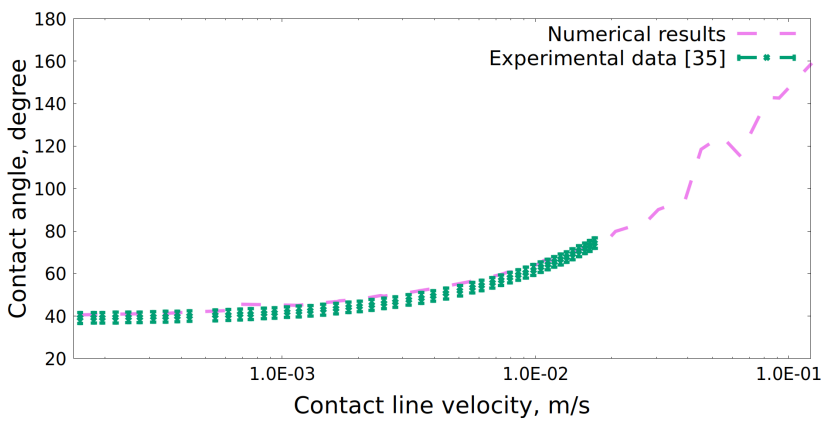

(a)

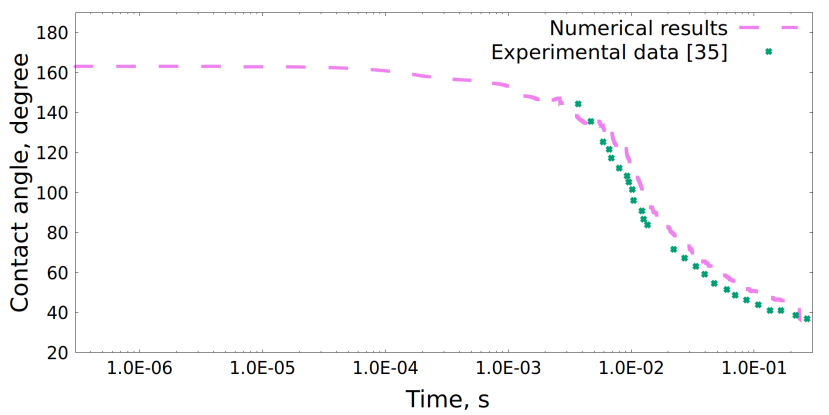

(b)

FIG. 13. Squalane droplet on silica: (a) contact angle evolution vs. contact line velocity (numerical vs. experimental), and (b) contact angle revolution vs. time (numerical vs. experimental).

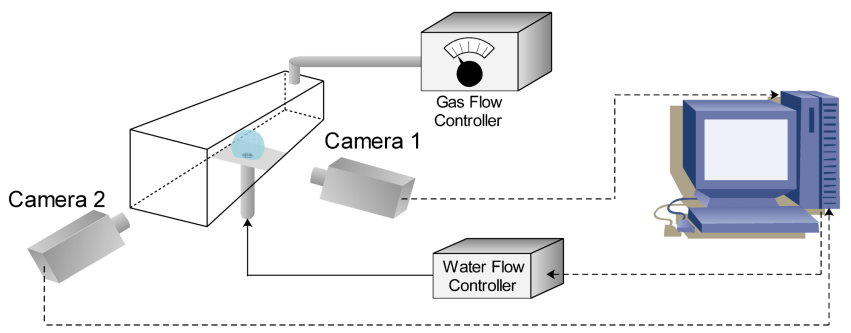

FIG. 14. Injection channel setup.

formed: i) water injection at a rate of $Q=5 \mu \mathrm{L} / \mathrm{s}$ on Kapton surface, ii) water injection at a rate of $Q=5 \mu \mathrm{L} / \mathrm{s}$ on PTFE, and iii) water injection at a rate of $Q=10 \mu \mathrm{L} / \mathrm{s}$ on PTFE. The injection profile is displayed in Fig 17. Two cameras, positioned perpendicular to each other, were used to capture the injection process as shown in Fig. 14 The spreading displacement were extracted from the recorded videos using built-in tracking feature of Image ${ }^{79}$, where the spreading regimes were identified by tracking the contact line every 0.2 second. The contact angle measurements were extracted using the "BSpline Snake" feature of the drop-analysis plugin of Image ${ }^{80}$. "B-Spline Snake" feature measures the contact angle locally following a concept of polynomial fit. Therefore, an error of $\pm 2.5^{\circ}$ was taken into account when measuring the contact angle, as shown in Fig. 15(b) and Fig. 16(b).

In order to perform the numerical simulations, an initial droplet configuration with a volume of $\approx 0.04$ and $\approx 0.2$ $\mu \mathrm{L}$ and initial contact angle of $75^{\circ}$ and $108^{\circ}$ for Kapton and PTFE, respectively, are selected. Fluid density, viscosity and surface tension coefficient are set to $\rho=1000 \mathrm{~kg} \mathrm{~m}^{-3}$, $\mu=8.90 \times 10^{-4} \mathrm{~kg} \mathrm{~m}^{-1} \mathrm{~s}^{-1}, \gamma=0.072 \mathrm{~N} \mathrm{~m}^{-1}$. Initial pressure in the liquid is set to $p_{0}=0 \mathrm{~Pa}$. The injection rates were of the order of micro seconds; hence, the average spreading displacement for this experiment was of the order $10^{-3} \mathrm{~m} \mathrm{~s}^{-1}$, as shown in Fig. 15(a) and Fig. 16(a), which yields $W e<1$. Accordingly, the capillary effect at the moving contact line is neglected compared to the dominant surface tension force. The time step and mesh size used for these simulations are chosen as $\Delta t=3 \times 10^{-3} \mathrm{~s}$ and $h=1.5 \times 10^{-4} \mathrm{~m}$, respectively.

\section{Kapton}

Fig. 15 shows the experimental and numerical results. Experimentally, the data was collected using videos instead of high speed capturing cameras, therefore, the spreading rate and contact angle evolution could not be tracked accurately at the initial stage, i.e., for $t<0.1 \mathrm{~s}$. Accordingly, a jump in the spreading radius and contact angle evolution was observed experimentally at the initial stage. Similarly, the initial injection rate causes an initial jump as well for the spreading radius for $t<0.1 \mathrm{~s}$.

It was observed experimentally and numerically that the spreading rate decreased as the droplet volume increased. Furthermore, the contact angle showed an average increase of $15^{\circ}$. This increase is interpreted in term of the work of adhesion. In general, the work of adhesion increases as the hydrophobicity of the surface decreases 81 . Accordingly, higher energy is required to detach or separate a liquid from hydrophilic surfaces due to the strong bonding between the liquid and the substrate 81 . During the initial stage of the injection process, this work of adhesion partially withstands the effect of the induced Young's stress as the droplet spreads towards its equilibrium contact angle. Hence, the contact angle increases as the injection process starts, i.e., from $75^{\circ}$ to $\sim 90^{\circ}$, as shown in Fig. 15(b), The maximum errors between the experimental data and the numerical results for both spreading rate and contact angle temporal evolution are estimated to be about $10 \%$, as shown in Fig. 15(a)

\section{PTFE}

Figure 16 shows the spreading radius and contact angle evolution as a function of time obtained experimentally and numerically. Experimental and numerical droplet radius are in excellent agreement at high injection flow rate, while at the lower flow rate the numerical results underpredict the spreading radius. The contact angle is nearly constant throughout both the experiment and simulation, as opposed to the case of kapton where there was a gradual increase in the contact angle. The average maximum errors of both injection rates was about $10 \%$. The contact angle evolution for both injection rates were in good agreement as well, with a maximum variation of $3^{\circ}$, i.e., about $2.5 \%$ (Fig. $16(\mathrm{~b})$ ). 


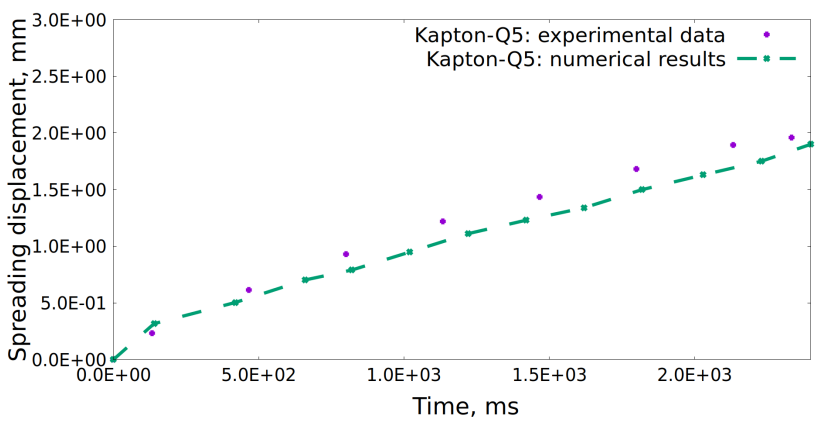

(a)

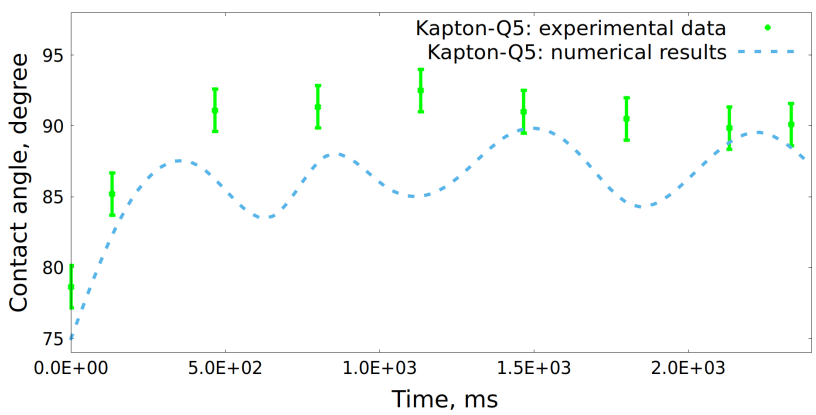

(b)

FIG. 15. Water-kapton injection analysis and comparison: (a), spreading displacement evolution, and (b) contact angle evolution.

As in the case of kapton, an initial jump was observed experimentally and numerically for the spreading radius for $t<20 \mathrm{~ms}$. Moreover, it is observed that the spreading rate decreased as the droplet volume increased. However, in the case of PTFE, the sessile droplet showed higher spreading rate at the initial stage due to the reduced work of adhesion between the liquid and substrate (hydrophobic substrate).

Fig. 17 shows a comparison between the experimental and simulated droplet profiles during injection. The model is capable of capturing the droplet profiles during the early stages of the process. After the droplet has grown substantially, predicted profiles show some disagreement with the experimental observations. It is hypothesized that the reason for the discrepancy is the lack of three dimensional information. The current 2D model neglects out-of-plane forces, which may play a significant role in determining the droplet shape and spreading rate $\sqrt{10}$.

\section{CONCLUSIONS}

A PFEM-based model for the simulation of droplet spreading on solid substrates was presented. Appropriate dynamic boundary conditions at the solid-liquid interface and the contact line were identified to provide physically meaningful results in droplet spreading simulations. A Navier-slip boundary condition is applied at the liquid-solid interface excluding the contact line. At the contact line, an effective slip coefficient was introduced as a function of capillary effects, and the

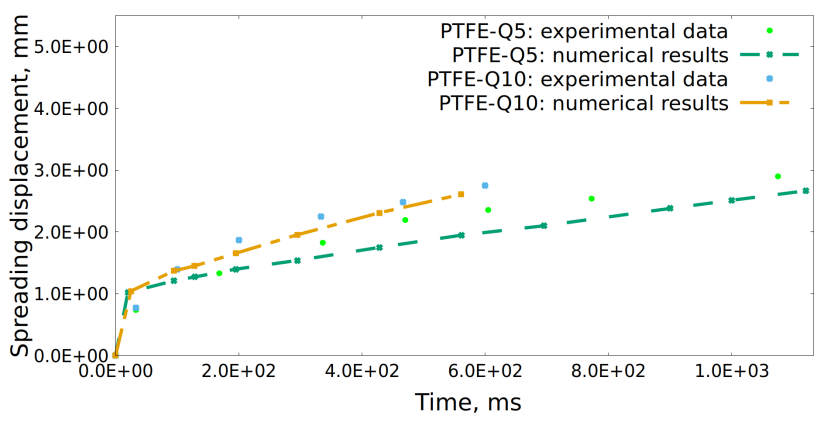

(a)

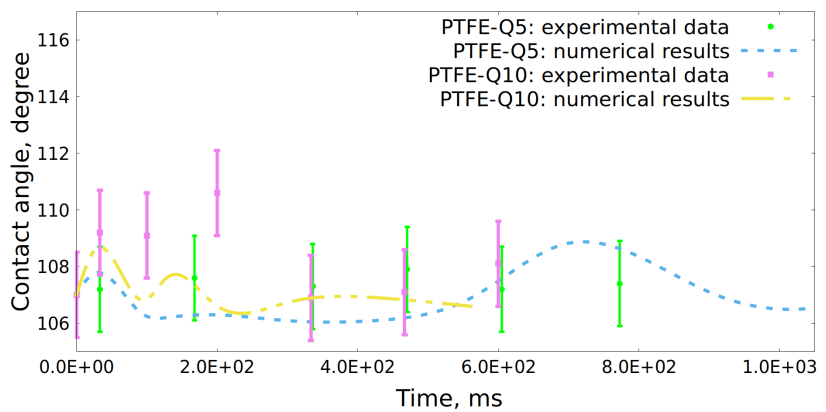

(b)

FIG. 16. Water-PTFE injection analysis and comparison: (a), spreading displacement evolution, and (b) contact angle evolution.
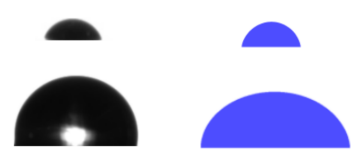

(a)

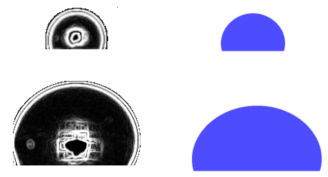

(b)

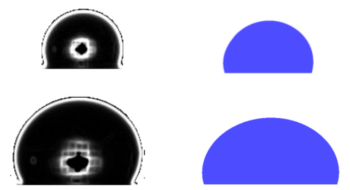

(c)
FIG. 17. Initial and final sessile droplet profiles for: (a) water injected at flow rate of $5 \mu \mathrm{L} / \mathrm{s}$ on kapton substrate, (b) water injected at flow rate of $5 \mu \mathrm{L} / \mathrm{s}$ on PTFE substrate, and (c) water injected at flow rate of $10 \mu \mathrm{L} / \mathrm{s}$ on PTFE substrate.

balance of stresses acting on the vicinity of the contact line.

The mesh size dependency of the solution was studied. First, the importance of the capillary wave propagation phenomena, and its numerical consequences in choosing the mesh size and time step were pointed out. Then, mesh-dependency studies were performed to show that the proposed dynamic 
boundary conditions alleviate the mesh-dependency of the solution.

The proposed droplet spreading model was validated by comparison with experimental results for a variety of liquids on hydrophobic and hydrophillic substrates. Spreading rates and contact angle temporal evolution obtained numerically were in good agreement with the experimental data. The model was able to capture the early spreading regime and the inertial to viscous transition regimes.

The model was compared with micro sessile-droplet injection experiments for water on hydrophilic and hydrophobic substrates. The experimental and numerical results were again in good agreement. A three-dimensional model must be developed in the future to account for out-of-plane geometric and physical effects for the simulation of spreading, injection and other interfacial dynamic phenomena.

\section{DATA AVAILABILITY STATEMENTS}

The data that support the findings of this study can be provided upon request.

\section{ACKNOWLEDGMENTS}

We are grateful to Drs. Howard Stone, James Bird, and Shreyas Mandre for their permission to use Fig. 11(a) published in reference 53 . We thank the Reviewers for their feedback and constructive comments. EM is thankful to Dr. Ajay Prasad for the fruitful discussion about the effect of shear stresses on droplet spreading phenomena.

\section{Compliance with ethical standards}

Funding EM acknowledges the financial support by Jubail University College and the Royal Commission for Jubail and Yanbu of Saudi Arabia. MS and AJ acknowledge financial support from the Natural Science and Engineering Research Council of Canada (NSERC) Collaborative Research and Development grant: NSERC CRDPJ 445887-12 and NSERC Discovery grant. PR was supported by AMADEUS project (reference: PGC2018-101655-B-I00) funded by the Spanish Ministry of Science, Innovation and Universities. TC and AZW acknowledge financial support by the Fuel Cell Performance and Durability Consortium (FC-PAD), by the Fuel Cell Technologies Office (FCTO), Office of Energy Efficiency and Renewable Energy (EERE), of the U.S. Department of Energy under contract number DE-AC02-05CH11231.

Conflict of interest The authors declare that they have no conflict of interest.

\section{APPENDIX A: ELEMENTAL CONTRIBUTION TO MATRIX ENTRIES}

Eqs.25 and 26 are assembled using the local matrices and vectors, with components defined as:

$$
\begin{aligned}
& M^{a b}=\rho \int_{\Omega_{X}} N^{a} N^{b} \mathrm{~d} \Omega_{\boldsymbol{X}}=\rho \int_{\Omega} N^{a} N^{b} J(\boldsymbol{X}) \mathrm{d} \Omega \\
& L^{a b}=\int_{\Omega_{X}} \frac{\partial N^{a}}{\partial X_{i}} \frac{\partial N^{b}}{\partial X_{i}} \Omega_{\boldsymbol{X}}=\int_{\Omega} \frac{\partial N^{a}}{\partial x_{i}} \frac{\partial N^{b}}{\partial x_{i}} J(\boldsymbol{X}) \mathrm{d} \Omega \\
& G_{i}^{a b}=-\int_{\Omega_{X}} \frac{\partial N^{a}}{\partial X_{i}} N^{b} d \Omega_{\boldsymbol{X}}=-\int_{\Omega} \frac{\partial N^{a}}{\partial x_{i}} N^{b} J(\boldsymbol{X}) \mathrm{d} \Omega \\
& f_{i}^{a}=\rho \int_{\Omega_{X}} N^{a} g_{i} d \Omega_{\boldsymbol{X}}=\rho \int_{\Omega} N^{a} g_{i} J(\boldsymbol{X}) \mathrm{d} \Omega \\
& D_{i}^{a b}=\int_{\Omega_{X}} N^{a} \frac{\partial N^{b}}{\partial X_{i}} d \Omega_{\boldsymbol{X}}=\int_{\Omega} N^{a} \frac{\partial N^{b}}{\partial x_{i}} J(\boldsymbol{X}) \mathrm{d} \Omega \\
& f_{s t, i}^{a}=-\int_{\Gamma_{I, \mathbf{X}}} \gamma \kappa N^{a} n_{i} d \Gamma_{\boldsymbol{X}}=-\int_{\Gamma_{I}} \gamma \kappa N^{a} n_{i} J_{\Gamma}(\boldsymbol{X}) \mathrm{d} \Gamma \\
& f_{\partial \Gamma_{, i}}^{a}=-\int_{\partial \Gamma_{\mathbf{X}}} \beta_{\partial \Gamma} u_{i} N^{a} d \partial \Gamma_{\boldsymbol{X}}=-\int_{\partial \Gamma} \beta_{\partial \Gamma} u_{i} N^{a} J_{\Gamma}(\boldsymbol{X}) \mathrm{d} \partial \Gamma \\
& f_{\Gamma_{S}, i}^{a}=-\int_{\Gamma_{S, \mathbf{X}}} \beta_{\Gamma_{s}} u_{i} N^{a} d \Gamma_{\boldsymbol{X}}=-\int_{\Gamma_{S}} \beta_{\Gamma_{s}} u_{i} N^{a} J_{\Gamma}(\boldsymbol{X}) \mathrm{d} \Gamma
\end{aligned}
$$

where $N^{a}$ represents the standard finite element shape function at node $a$, and the index $i$ is used for the spatial components. The present model is based on the updated Lagrangian formulation (ULF), and therefore the integration domains in Eqs. A1]- A5, $\Omega_{X}$, correspond to the updated configuration. The transformation between the reference configuration, $\Omega$, and the updated one is performed using the Jacobians $J(\boldsymbol{X})$ and $J_{\Gamma}(\boldsymbol{X})$.

\section{APPENDIX B: SOLUTION ALGORITHM}

Given a known configuration $\boldsymbol{X}_{n}$, velocity $\overline{\boldsymbol{v}}_{n}$, and pressure $\bar{p}_{n}$, at time $t_{n}$, the procedure for obtaining the values of these variables at the next time step $t_{n+1}$ is summarized in Algorithm 1 A complete general algorithm, including a two-phase flow model, can be found in reference 37 .

\section{APPENDIX C: IMPLEMENTATION}

The numerical method was implemented within Kratos MultiPhysics version 6, a C++ object oriented FE open-source framework 82 . For droplet spreading, the experimental data found in reference ${ }^{35[53]}$ was extracted using WebPlotDigitizer software $83[84$.

\section{REFERENCES}

\footnotetext{
${ }^{1}$ S. Shukla, K. Domican, K. Karan, S. Bhattacharjee, and M. Secanell, "Analysis of low platinum loading thin polymer electrolyte fuel cell electrodes prepared by inkjet printing," Electrochimica Acta 156, 289-300 (2015).

${ }^{2} \mathrm{~F}$. Fu, P. Li, K. Wang, and R. Wu, "Numerical simulation of sessile droplet spreading and penetration on porous substrates," Langmuir 35, 2917-2924 (2019).

${ }^{3}$ A. Klimanek, "Numerical modelling of natural draft wet-cooling towers," Archives of Computational Methods in Engineering 20, 61-109 (2013).
} 


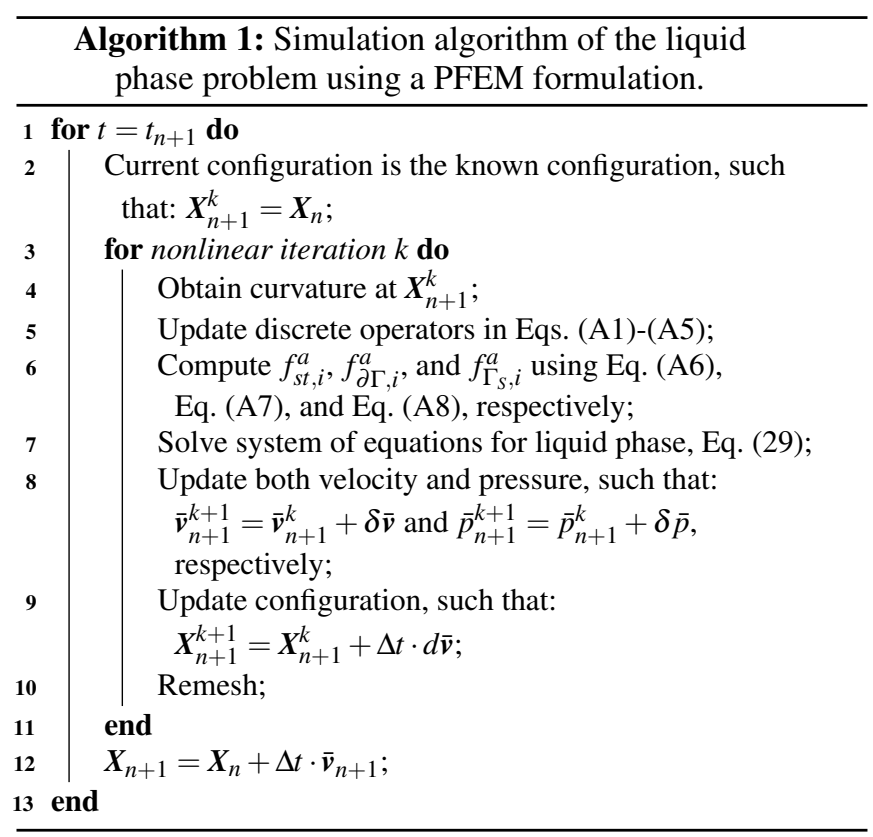

${ }^{4}$ P. García-Salaberri, I. Zenyuk, G. Hwang, M. Vera, A. Weber, and J. Gostick, "Implications of inherent inhomogeneities in thin carbon fiber-based gas diffusion layers: A comparative modeling study," Electrochimica Acta 295, 861-874 (2019).

${ }^{5}$ M. Sabharwal, J. Gostick, and M. Secanell, "Virtual liquid water intrusion in fuel cell gas diffusion media," Journal of The Electrochemical Society 165, F553-F563 (2018).

${ }^{6}$ M. Secanell, A. Jarauta, A. Kosakian, M. Sabharwal, and J. Zhou, "PEM fuel cells, modeling," in Encyclopedia of Sustainability Science and Technology, edited by R. A. Meyers (Springer New York, New York, NY, 2017) pp. $1-61$.

${ }^{7}$ J. Zhou, D. Stanier, A. Putz, and M. Secanell, "A mixed wettability pore size distribution based mathematical model for analyzing two-phase flow in porous electrodes II. Model validation and analysis of micro-structural parameters," Journal of The Electrochemical Society 164, F540-F556 (2017).

${ }^{8}$ M. Andersson, V. Vukčević, S. Zhang, Y. Qi, H. Jasak, S. Beale, and W. Lehnert, "Modeling of droplet detachment using dynamic contact angles in polymer electrolyte fuel cell gas channels," International Journal of Hydrogen Energy 44, 11088-11096 (2019).

${ }^{9}$ A. Jarauta, P. Ryzhakov, M. Secanell, P. Waghmare, and J. Pons-Prats, "Numerical study of droplet dynamics in a polymer electrolyte fuel cell gas channel using an embedded Eulerian-Lagrangian approach," Journal of Power Sources 323, 201-212 (2016).

${ }^{10}$ P. Ryzhakov, A. Jarauta, M. Secanell, and J. Pons-Prats, "On the application of the PFEM to droplet dynamics modeling in fuel cells," Computational Particle Mechanics 4, 285-295 (2017).

${ }^{11}$ A. Santamaria, P. Das, J. MacDonald, and A. Weber, "Liquid-water interactions with gas-diffusion-layer surfaces," Journal of The Electrochemical Society 161, F1184-F1193 (2014).

${ }^{12}$ A. Jarauta and P. Ryzhakov, "Challenges in Computational Modeling of Two-Phase Transport in Polymer Electrolyte Fuel Cells Flow Channels: A Review," Archives of Computational Methods in Engineering 25, 10271057 (2018).

${ }^{13} \mathrm{M}$. Wörner, "Numerical modeling of multiphase flows in microfluidics and micro process engineering: a review of methods and applications," Microfluidics and nanofluidics 12, 841-886 (2012).

${ }^{14} \mathrm{~J}$. Venkatesan and S. Ganesan, "Computational modeling of impinging viscoelastic droplets," Journal of Non-Newtonian Fluid Mechanics 263, 42-60 (2019).

${ }^{15} \mathrm{~W}$. Ren and W. E, "Boundary conditions for the moving contact line problem," Physics of fluids 19, 022101 (2007).

${ }^{16} \mathrm{~J}$. Venkatesan and S. Ganesan, "On the Navier-slip boundary condition for computations of impinging droplets," in 2015 IEEE 22nd International
Conference on High Performance Computing Workshops (IEEE, 2015) pp. $2-11$.

${ }^{17}$ J. Zhang, M. K. Borg, and J. M. Reese, "Multiscale simulation of dynamic wetting," International Journal of Heat and Mass Transfer 115, 886-896 (2017).

${ }^{18}$ A. M. Boelens and J. J. de Pablo, "Generalised Navier boundary condition for a volume of fluid approach using a finite-volume method," Physics of Fluids 31, 021203 (2019).

${ }^{19}$ L. Chen, E. Bonaccurso, and M. E. Shanahan, "Inertial to viscoelastic transition in early drop spreading on soft surfaces," Langmuir 29, 1893-1898 (2013).

${ }^{20} \mathrm{G}$. Kuznetsov, D. Feoktistov, and E. Orlova, "Regimes of spreading of a water droplet over substrates with varying wettability," Journal of Engineering Physics and Thermophysics 89, 317-322 (2016).

${ }^{21}$ S. Afkhami, S. Zaleski, and M. Bussmann, "A mesh-dependent model for applying dynamic contact angles to VOF simulations," Journal of computational physics 228, 5370-5389 (2009).

${ }^{22} \mathrm{G}$. Buscaglia and R. Ausas, "Variational formulations for surface tension, capillarity and wetting," Computer Methods in Applied Mechanics and Engineering 200, 3011-3025 (2011).

${ }^{23} \mathrm{~K}$. J. Vachaparambil and K. E. Einarsrud, "Comparison of surface tension models for the volume of fluid method," Processes 7, 542 (2019).

${ }^{24}$ T. Yamamoto, Y. Okano, and S. Dost, "Validation of the S-CLSVOF method with the density-scaled balanced continuum surface force model in multiphase systems coupled with thermocapillary flows," International Journal for Numerical Methods in Fluids 83, 223-244 (2017).

${ }^{25}$ M. Eres, L. Schwartz, and R. Roy, "Fingering phenomena for driven coating films," Physics of Fluids 12, 1278-1295 (2000).

${ }^{26}$ D. Bonn, J. Eggers, J. Indekeu, J. Meunier, and E. Rolley, "Wetting and spreading," Reviews of modern physics 81, 739 (2009).

${ }^{27} \mathrm{E}$. Dussan, "On the spreading of liquids on solid surfaces: static and dynamic contact lines," Annual Review of Fluid Mechanics 11, 371-400 (1979).

${ }^{28} \mathrm{C}$. Huh and L. Scriven, "Hydrodynamic model of steady movement of a solid/liquid/fluid contact line," Journal of colloid and interface science $\mathbf{3 5}$, 85-101 (1971).

${ }^{29}$ Y. D. Shikhmurzaev, "Singularities at the moving contact line. Mathematical, physical and computational aspects," Physica D: Nonlinear Phenomena 217, 121-133 (2006).

${ }^{30}$ S. Manservisi and R. Scardovelli, "A variational approach to the contact angle dynamics of spreading droplets," Computers \& Fluids 38, 406-424 (2009).

${ }^{31}$ S. C. Cho, Y. Wang, and K. Chen, "Droplet dynamics in a polymer electrolyte fuel cell gas flow channel: Forces, deformation and detachment. II: Comparisons of analytical solution with numerical and experimental results," Journal of Power Sources 210, 191-197 (2012).

${ }^{32}$ A. Theodorakakos, T. Ous, M. Gavaises, J. Nouri, N. Nikolopoulos, and H. Yanagihara, "Dynamics of water droplets detached from porous surfaces of relevance to PEM fuel cells," Journal of Colloid and Interface Science 300, 673-687 (2006).

${ }^{33}$ X. Zhu, Q. Liao, P. Sui, and N. Djilali, "Numerical investigation of water droplet dynamics in a low-temperature fuel cell microchannel: Effect of channel geometry," Journal of Power Sources 195, 801-812 (2010).

${ }^{34}$ A. Jarauta, P. Ryzhakov, J. Pons-Prats, and M. Secanell, "An implicit surface tension model for the analysis of droplet dynamics," Journal of Computational Physics 374, 1196-1218 (2018).

${ }^{35}$ D. Seveno, A. Vaillant, R. Rioboo, H. Adao, J. Conti, and J. D. Coninck, "Dynamics of wetting revisited," Langmuir 25, 13034-13044 (2009).

${ }^{36} \mathrm{P}$. Spelt, "A level-set approach for simulations of flows with multiple moving contact lines with hysteresis," Journal of Computational Physics 207, 389-404 (2005)

${ }^{37}$ P. Ryzhakov and A. Jarauta, "An embedded approach for immiscible multifluid problems," International Journal for Numerical Methods in Fluids 81, 357-376 (2016).

${ }^{38}$ E. Oñate, S. Idelsohn, F. D. Pin, and R. Aubry, "The particle finite element method: an overview," International Journal of Computational Methods 1, 267-307 (2004)

${ }^{39}$ S. R. Idelsohn, E. Oñate, and F. D. Pin, "The particle finite element method: a powerful tool to solve incompressible flows with free-surfaces and breaking waves," International journal for numerical methods in engineering 61, 
964-989 (2004)

${ }^{40}$ J. T. Davies, Interfacial phenomena (Elsevier, 2012).

${ }^{41}$ R. Bird, W. Stewart, and E. Lightfoot, Transport Phenomena, 2nd ed. (John Wiley and Sons, 2002).

${ }^{42}$ F. White, Viscous Fluid Flow, 2nd ed. (McGraw-Hill, 1991).

${ }^{43}$ P. Ryzhakov, R. Rossi, S. Idelsohn, and E. Oñate, "A monolithic lagrangian approach for fluid-structure interaction problems," Computational Mechanics 46, 883-899 (2010).

${ }^{44}$ C. Navier, "Mémoire sur les lois du mouvement des fluides," Mémoires de l'Académie Royale des Sciences de l'Institut de France 6, 389-440 (1823).

${ }^{45}$ Y. Yoon, D. Kim, and J.-B. Lee, "Hierarchical micro/nano structures for super-hydrophobic surfaces and super-lyophobic surface against liquid metal," Micro and Nano Systems Letters 2, 3 (2014).

${ }^{46}$ Y. Yuan and T. R. Lee, "Contact angle and wetting properties," in Surface science techniques (Springer, 2013) pp. 3-34.

${ }^{47}$ A. Jarauta, M. Secanell, J. Pons-Prats, P. Ryzhakov, S. Idelsohn, and E. Oñate, "A semi-analytical model for droplet dynamics on the GDL surface of a PEFC electrode," international journal of hydrogen energy 40, 5375-5383 (2015).

${ }^{48}$ I. S. Bayer and C. M. Megaridis, "Contact angle dynamics in droplets impacting on flat surfaces with different wetting characteristics," Journal of Fluid Mechanics 558, 415-449 (2006).

${ }^{49}$ T.-S. Jiang, O. Soo-Gun, and J. C. Slattery, "Correlation for dynamic contact angle,” Journal of Colloid and Interface Science 69, 74-77 (1979).

${ }^{50} \mathrm{M}$. Bracke, F. De Voeght, and P. Joos, "The kinetics of wetting: the dynamic contact angle," in Trends in Colloid and Interface Science III (Springer, 1989) pp. 142-149.

${ }^{51}$ J. E. Seebergh and J. C. Berg, "Dynamic wetting in the low capillary number regime,” Chemical Engineering Science 47, 4455-4464 (1992).

${ }^{52}$ S. Van Mourik, A. Veldman, and M. Dreyer, "Simulation of capillary flow with a dynamic contact angle," Microgravity-Science and Technology 17, 87 (2005).

${ }^{53}$ J. C. Bird, S. Mandre, and H. A. Stone, "Short-time dynamics of partial wetting," Physical review letters 100, 234501 (2008).

${ }^{54}$ J. B. Keller, P. A. Milewski, and J.-M. Vanden-Broeck, "Merging and wetting driven by surface tension," European Journal of Mechanics-B/Fluids 19, 491-502 (2000).

${ }^{55}$ L. Chen, G. K. Auernhammer, and E. Bonaccurso, "Short time wetting dynamics on soft surfaces," Soft Matter 7, 9084-9089 (2011).

${ }^{56}$ M.-J. Wang, F.-H. Lin, Y.-L. Hung, and S.-Y. Lin, "Dynamic behaviors of droplet impact and spreading: water on five different substrates," Langmuir 25, 6772-6780 (2009).

${ }^{57}$ Y. Renardy, S. Popinet, L. Duchemin, M. Renardy, S. Zaleski, C. Josserand, M. Drumright-Clarke, D. Richard, C. Clanet, and D. Quéré, "Pyramidal and toroidal water drops after impact on a solid surface," Journal of Fluid Mechanics 484, 69-83 (2003).

${ }^{58}$ P. Li, S. Wang, and W. Dong, "Capillary wave and initial spreading velocity at impact of drop onto a surface." Journal of Applied Fluid Mechanics 12 (2019).

${ }^{59}$ F. Denner and B. G. van Wachem, "Numerical time-step restrictions as a result of capillary waves," Journal of Computational Physics 285, 24-40 (2015).

${ }^{60} \mathrm{~F}$. Durst, Fluid mechanics: an introduction to the theory of fluid flows (Springer Science \& Business Media, 2008).

${ }^{61}$ P. Ryzhakov, E. Oñate, R. Rossi, and S. Idelsohn, Lagrangian FE methods for coupled problems in fluid mechanics (CIMNE, 2010) p. 199.

${ }^{62}$ P. Ryzhakov, J. Marti, S. Idelsohn, and E. Oñate, "Fast fluid-structure interaction simulations using a displacement-based finite element model equipped with an explicit streamline integration prediction," Computer Methods in Applied Mechanics and Engineering 315, 1080-1097 (2017).

${ }^{63}$ S. Idelsohn, E. Oñate, and F. del Pin, "The particle finite element method: a powerful tool to solve incompressible flows with free-surfaces and breaking waves," International Journal for Numerical Methods in Engineering 61,
964-989 (2004).

${ }^{64}$ S. Idelsohn, J. Marti, A. Souto-Iglesias, and E. Oñate, "Interaction between an elastic structure and free-surface flows: experimental versus numerical comparisons using the PFEM,' Computational Mechanics 43, 125-132 (2008).

${ }^{65}$ E. Oñate, S. R. Idelsohn, M. A. Celigueta, R. Rossi, J. Marti, J. M. Carbonell, P. Ryzhakov, and B. Suárez, "Advances in the particle finite element method (PFEM) for solving coupled problems in engineering," in Particle-Based Methods (Springer, 2011) pp. 1-49.

${ }^{66}$ J. Marti and P. Ryzhakov, "An explicit-implicit finite element model for the numerical solution of incompressible navier-stokes equations on moving grids," Computer Methods in Applied Mechanics and Engineering 350, 750-765 (2019)

${ }^{67}$ J. Marti and P. Ryzhakov, "An explicit/implicit Runge-Kutta-based PFEM model for the simulation of thermally coupled incompressible flows," Computational Particle Mechanics 7, 57-69 (2020).

${ }^{68} \mathrm{~J}$. Donea and A. Huerta, Finite Element Methods for Flow Problems, 1 st ed. (John Wiley \& Sons, 2003).

${ }^{69}$ R. Codina, "A stabilized finite element method for generalized stationary incompressible flows," Computer Methods in Applied Mechanics and Engineering 190, 2681 - 2706 (2001)

${ }^{10}$ E. Moreno, A. Larese, and M. Cervera, "Modelling of Bingham and Herschel-Bulkley flows with mixed P1/P1 finite elements stabilized with orthogonal subgrid scale," Journal of Non-Newtonian Fluid Mechanics 228, 1-16 (2016).

${ }^{71}$ M. Chowdhury and B. R. Kumar, "On subgrid multiscale stabilized finite element method for advection-diffusion-reaction equation with variable coefficients," Applied Numerical Mathematics 150, 576-586 (2020).

${ }^{72}$ M. Renardy, Y. Renardy, and J. Li, "Numerical simulation of moving contact line problems using a volume-of-fluid method," Journal of Computational Physics 171, 243-263 (2001).

${ }^{73}$ D. K. Owens and R. Wendt, "Estimation of the surface free energy of polymers," Journal of applied polymer science 13, 1741-1747 (1969).

${ }^{74}$ F. D. Egitto and L. J. Matienzo, "Plasma modification of polymer surfaces for adhesion improvement," IBM Journal of Research and Development 38, 423-439 (1994)

${ }^{75} \mathrm{~L}$. Chen, Y. He, and W. Tao, "Effects of surface microstructures of gas diffusion layer on water droplet dynamic behaviors in a micro gas channel of proton exchange membrane fuel cells," International Journal of Heat and Mass Transfer 60, 252-262 (2013).

${ }^{76}$ L. Courbin, J. C. Bird, M. Reyssat, and H. A. Stone, "Dynamics of wetting: from inertial spreading to viscous imbibition," Journal of Physics: Condensed Matter 21, 464127 (2009).

${ }^{77}$ H. Ding and P. D. Spelt, "Inertial effects in droplet spreading: a comparison between diffuse-interface and level-set simulations," Journal of fluid mechanics 576, 287-296 (2007).

${ }^{78}$ A.-L. Biance, C. Clanet, and D. Quéré, "First steps in the spreading of a liquid droplet," Physical Review E 69, 016301 (2004).

${ }^{79}$ C. Schneider, W. Rasband, and K. Eliceiri, "NIH Image to ImageJ: 25 years of image analysis," Nature methods 9, 671 (2012).

${ }^{80}$ A. Stalder, G. Kulik, D. Sage, L. Barbieri, and P. Hoffmann, "A snakebased approach to accurate determination of both contact points and contact angles," Colloids And Surfaces A: Physicochemical And Engineering Aspects 286, 92-103 (2006).

${ }^{81}$ M.-O. Samuelsson and D. L. Kirchman, "Degradation of adsorbed protein by attached bacteria in relationship to surface hydrophobicity," Appl. Environ. Microbiol. 56, 3643-3648 (1990).

${ }^{82}$ P. Dadvand, R. Rossi, and E. Oñate, "An object-oriented environment for developing finite element codes for multi-disciplinary applications," Archieves of Computational Methods in Engineering 17/3, 253-297 (2010).

${ }^{83}$ A. Rohatgi, "Webplotdigitizer," (2011).

${ }^{84}$ J. Marti, P. Ryzhakov, S. Idelsohn, and E. Oñate, "Combined eulerianPFEM approach for analysis of polymers in fire situations," International Journal for Numerical Methods in Engineering 92, 782-801 (2012). 